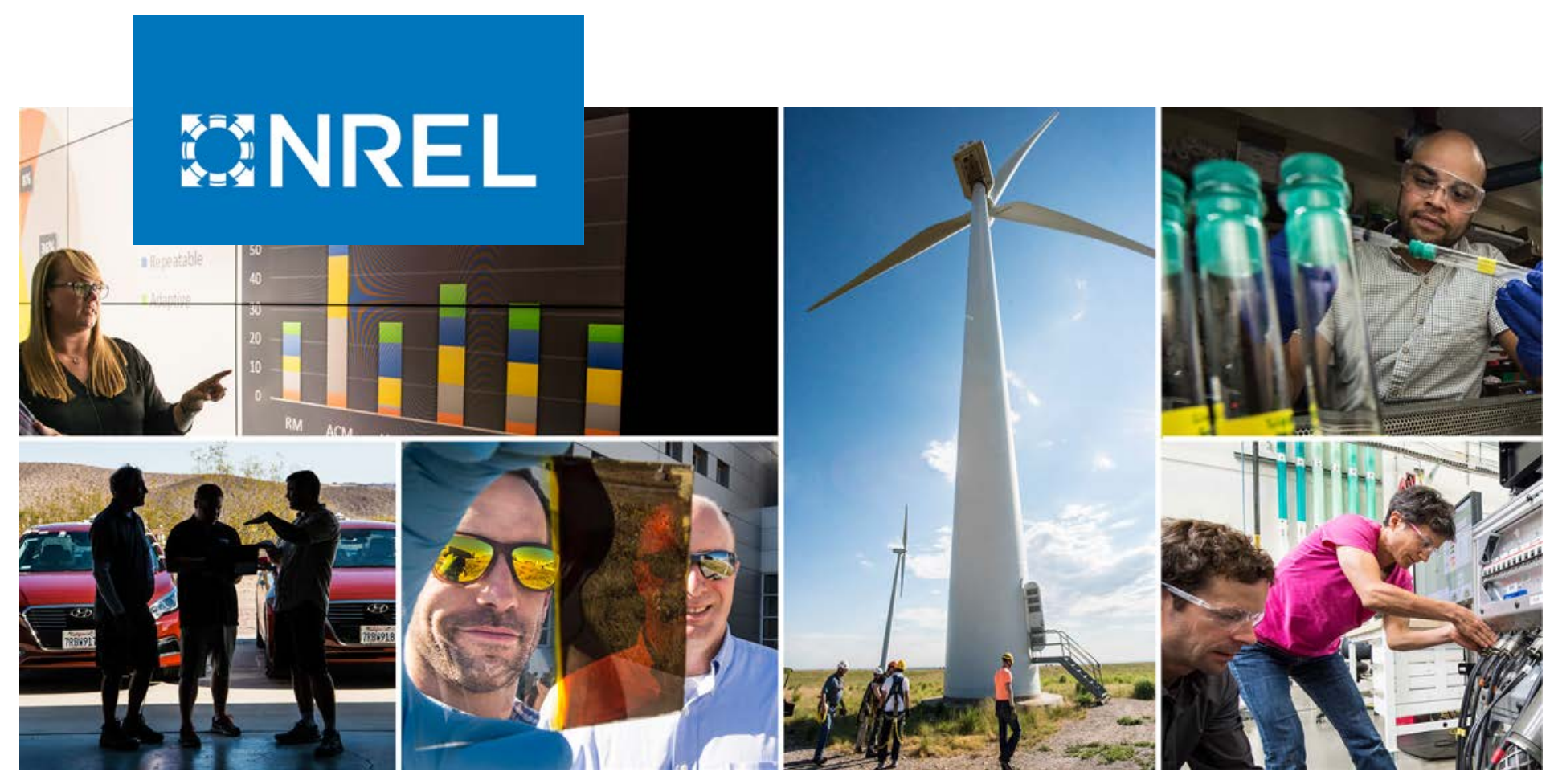

\title{
Evaluating the Impact of Residential Solar Contract Cancellations in the United States
}

Jesse R. Cruce, ${ }^{1}$ Eric O'Shaughnessy, ${ }^{2}$ and Jeffrey J. Cook ${ }^{1}$

1 National Renewable Energy Laboratory

2 Clean Kilowatts, LLC

NREL is a national laboratory of the U.S. Department of Energy

Office of Energy Efficiency \& Renewable Energy

Operated by the Alliance for Sustainable Energy, LLC

This report is available at no cost from the National Renewable Energy Laboratory (NREL) at www.nrel.gov/publications.
Technical Report

NREL/TP-6A20-80626

January 2022 


\title{
GNREL
}

\section{Evaluating the Impact of Residential Solar Contract Cancellations in the United States}

\author{
Jesse R. Cruce, ${ }^{1}$ Eric O'Shaughnessy, ${ }^{2}$ and \\ Jeffrey J. Cook ${ }^{1}$
}

1 National Renewable Energy Laboratory

2 Clean Kilowatts, LLC

\section{Suggested Citation}

Cruce, Jesse R., Eric O'Shaughnessy, and Jeffrey J. Cook. 2022. Evaluating the Impact of Residential Contract Cancellations in the United States. Golden, CO: National Renewable Energy Laboratory. NREL/TP-6A20-80626.

https://www.nrel.gov/docs/fy22osti/80626.pdf.

NREL is a national laboratory of the U.S. Department of Energy Office of Energy Efficiency \& Renewable Energy Operated by the Alliance for Sustainable Energy, LLC

This report is available at no cost from the National Renewable Energy Laboratory (NREL) at www.nrel.gov/publications.

Contract No. DE-AC36-08GO28308
Technical Report

NREL/TP-6A20-80626

January 2022

National Renewable Energy Laboratory 15013 Denver West Parkway Golden, CO 80401

303-275-3000 • www.nrel.gov 


\section{NOTICE}

This work was authored in part by the National Renewable Energy Laboratory, operated by Alliance for Sustainable Energy, LLC, for the U.S. Department of Energy (DOE) under Contract No. DE-AC36-08GO28308. Funding provided by the U.S. Department of Energy Office of Energy Efficiency and Renewable Energy Solar Energy Technologies Office. The views expressed herein do not necessarily represent the views of the DOE or the U.S. Government.

This report is available at no cost from the National Renewable Energy Laboratory (NREL) at www.nrel.gov/publications.

U.S. Department of Energy (DOE) reports produced after 1991 and a growing number of pre-1991 documents are available free via www.OSTI.gov.

Cover Photos by Dennis Schroeder: (clockwise, left to right) NREL 51934, NREL 45897, NREL 42160, NREL 45891, NREL 48097, NREL 46526.

NREL prints on paper that contains recycled content. 


\section{Acknowledgments}

This work was supported by the Solar Energy Technologies Office at the U.S. Department of Energy. The authors would like to thank the following individuals and organizations for their thoughtful review of this work: Valerie Anderson (Tesla), Karyn Boenker (Sunrun), David Feldman (NREL), Eric Jung (Titan Solar Power), Steve Letendre (Freedom Forever), Ammar Qusaibaty (SETO), Vignesh Ramasamy (NREL), Abigail Randall (SETO), Christopher Sabin (GenRenew), and Dara Yung (Sunrun). 


\section{List of Acronyms}

ATB

AHJ

CSI

IX

approval to build

authority having jurisdiction

California Solar Initiative

NREL

P/ATB

interconnection

PII

National Renewable Energy Laboratory

permit/approval to build

PTO

permitting, inspection, and interconnection

PV

permission to operate

SolarTRACE

(solar) photovoltaic

Solar Time-Based Residential Analytics and Cycle Time Estimator 


\section{Executive Summary}

The residential solar photovoltaic (PV) market in the United States has grown rapidly in the last decade, from less than 50,000 systems installed in 2010 to over 420,000 systems in 2020 (Davis et al., 2021). The process for installing residential PV systems includes distinct phases and costs for customer acquisition, contract negotiation and signing, system design, and permitting and interconnection applications to the local city or county and utility. Most customers who sign contracts successfully install PV systems (Cook et al., 2021; Liao, 2020); however, some customers instead choose to terminate or cancel their contracts prior to install. These contract cancellations may result in lost time and higher "soft costs" (non-equipment costs) that must be borne by the installer. Depending on installer practices, higher soft costs for installers may ultimately contribute to higher prices for customers who successfully install PV systems. To date, few studies have attempted to estimate cancellation rates or assess their impacts on installed residential PV system costs.

Customer decisions to cancel contracts or purchases are common across many consumer industries. Many states have consumer protection laws allowing "cooling off periods" for customers to cancel signed contracts without penalties. Beyond these laws, PV installer practices and contract terms vary. Some installers allow penalty-free cancellations up until install, while others may charge cancellation fees out of down payments or deposits. Contract terms may also differ depending on the type of contract and ownership model (i.e., customer-owned system, leased, or third-party owned). Similarly, customer contract cancellation rates (and subsequent costs) likely vary widely between installers, with installer size and business practices likely playing a role, along with other factors such as incentive programs, system costs, project timelines, and the permitting/interconnection process (Cook et al., 2021).

In this report, we utilize a project-level data set of 199,665 residential PV-only contracts from four medium-to-large installers collected for NREL's Solar Time-Based Residential Analytics and Cycle Time Estimator (SolarTRACE) (NREL, 2021). Installers of this size install at least 100 (medium installers) or at least 1,000 (large installers) systems per year. Together the installers in this data set cover about 10\% of U.S. installs each year from 2017-2019 (based on Davis et al. (2021)). With this data, we evaluate cancellation rates and trends from contract signing to install. Additional data collected for SolarTRACE also allowed for cancellation rate comparisons with installers altogether covering $22 \%-34 \%$ of U.S. residential installs across these three years. Finally, we estimate installer spending at each phase of the process and calculate the potential cost impact of contract cancellations.

\section{Cancellation Rates and Trends}

Figure ES-1 summarizes the cancellation rates and trends in this data set. We found that $51 \%$ of contracts in this data set did not result in an installed PV system, although the median installer cancellation rate is somewhat lower (33\%). The SolarTRACE data suggests cancellation rates for those additional installers were similarly within this range. The cancellation rates in this study were significantly higher than previous estimates of 11\%-12\% (Cook et al., 2021; Liao, 2020). One explanation is that the installers in this study are generally much larger than the majority of U.S. solar companies, and smaller installers have typically reported lower cancellation rates than larger installers (Cook et al., 2021). While overall nationwide cancellation rates cannot be 
estimated from this study, our results suggest that at least a quarter of U.S. installs during these years were performed by companies with higher cancellation rates than previous estimates.

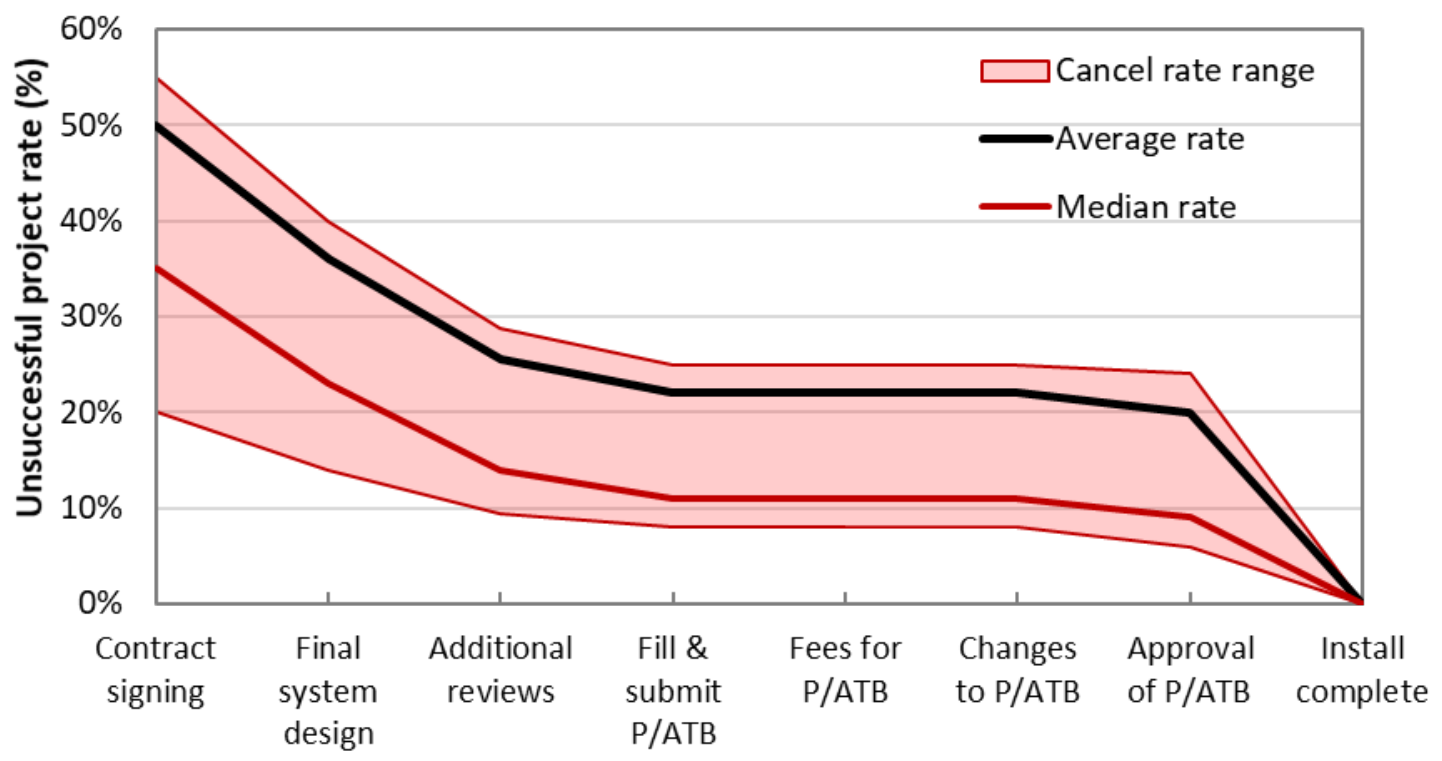

Figure ES-1. Cancellation rates for the post-contract/pre-install phases identified by this study. The average rate is presented for the entire project-level data set, whereas the median and range come from rates across individual installers and years (2017-2019) within the data set. (PIATB means permit/approval to build application.)

We find that the majority (73\%) of contract cancellations happen prior to permit and/or approval to build (ATB) interconnection application submission. However, 10\%-20\% of projects with an approved permit and/or ATB also do not install systems. On the other hand, cancellations during the permit/ATB review process (between submission and approval) are rare, accounting for only $2 \%$ of unsuccessful projects in the data. No significant timeline differences between installed and unsuccessful projects were found in the data, either between contract signing and permit/ATB submission or in permit/ATB review timelines. These findings, along with a review of the existing literature, suggest there is no single driver of PV contract cancellations, though customer decisions, experiences, and finances, the cost/benefit proposition, and the permitting process all likely play a role.

\section{Installer Soft Cost Model}

Figure ES-2 shows the accumulated installer spending as an individual project progresses through each of the pre-install phases. This model provides a benchmark for estimating the lost spending on an individual project when it is cancelled at a given phase (assuming the installer does not require and/or keep a deposit from the customer). The average and range of costs are shown on a per-project basis, while the net cost per install represents overall soft costs for successful projects, taken directly from Feldman et al. (2021). 


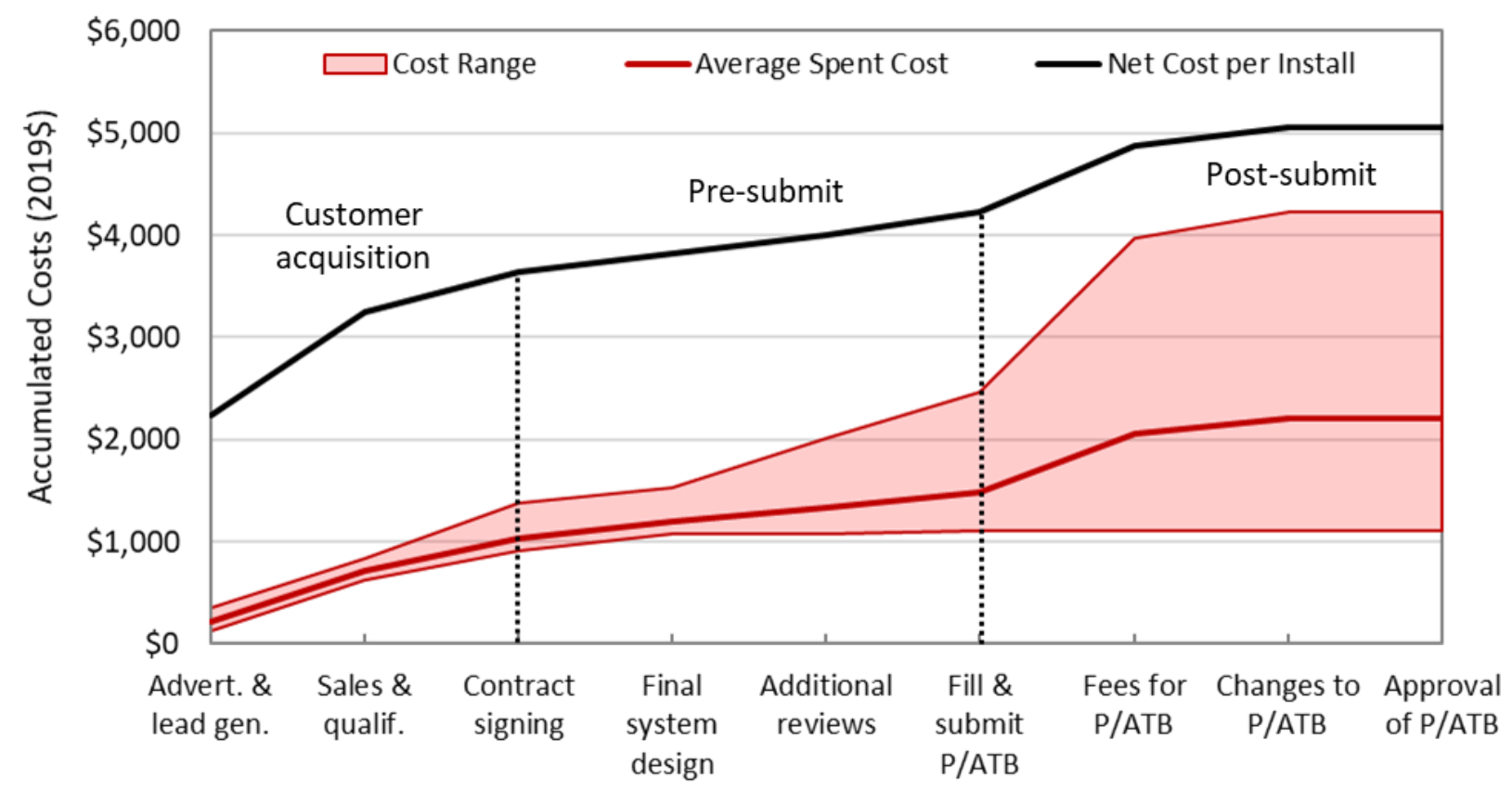

Figure ES-2. Accumulated installer spending/costs through each phase of the pre-install process. The average and range of costs are based on direct installer spending on a per-project basis, whereas net costs per install include the higher soft costs resulting from spending on unsuccessful projects. Only projects with signed contracts are considered cancellations. Net costs are taken directly from Feldman et al. (2021).

As seen in Figure ES-2, direct per-project spending on customer acquisition averages around $\$ 1,000$ by contract signing. Costs diverge significantly from there, depending on the costs associated with the authority having jurisdiction (AHJ) permitting and utility ATB requirements. Average spending by permit/ATB approval is around $\$ 2,000$ per project, though costs may be as high as $\$ 4,000$ or more for higher-cost jurisdictions (from high application fees, more costly inperson submission methods, required professional engineering reviews, etc.). Net costs per install are generally much higher than average per-project spending due to low install success rates for spending in early phases - in this model, all lost spending on unsuccessful projects is borne by successful installs, hence the higher overall net costs.

\section{Cancellation Cost Impacts}

Figure ES-3 shows the impact of contract cancellations on net soft costs for installed projects. On average, contract cancellations (when they occur) may add about $\$ 0.20 / \mathrm{W}$ to soft costs, or about $25 \%$ of direct pre-install soft costs (which average about $\$ 0.75 / \mathrm{W}$ ). ${ }^{1}$ However, there is significant variation in the possible results, with cost impacts potentially ranging from $\$ 0.04$ $\$ 0.40 / \mathrm{W}$ (median $\$ 0.10 / \mathrm{W}$ ). Most of the impact comes from customer acquisition spending that is lost in early phase cancellations. Again, whether or how much of these costs are passed on to successful PV customers versus being absorbed by the installer likely varies with installer practices, contract terms, and their volume of cancellations.

\footnotetext{
${ }^{1}$ Excluding overhead, install labor, taxes, installer margins, and other indirect costs, which altogether bring soft costs up to about $\$ 1.5 / \mathrm{W}$ (around $\$ 10,500$ per project) (Feldman et al., 2021).
} 


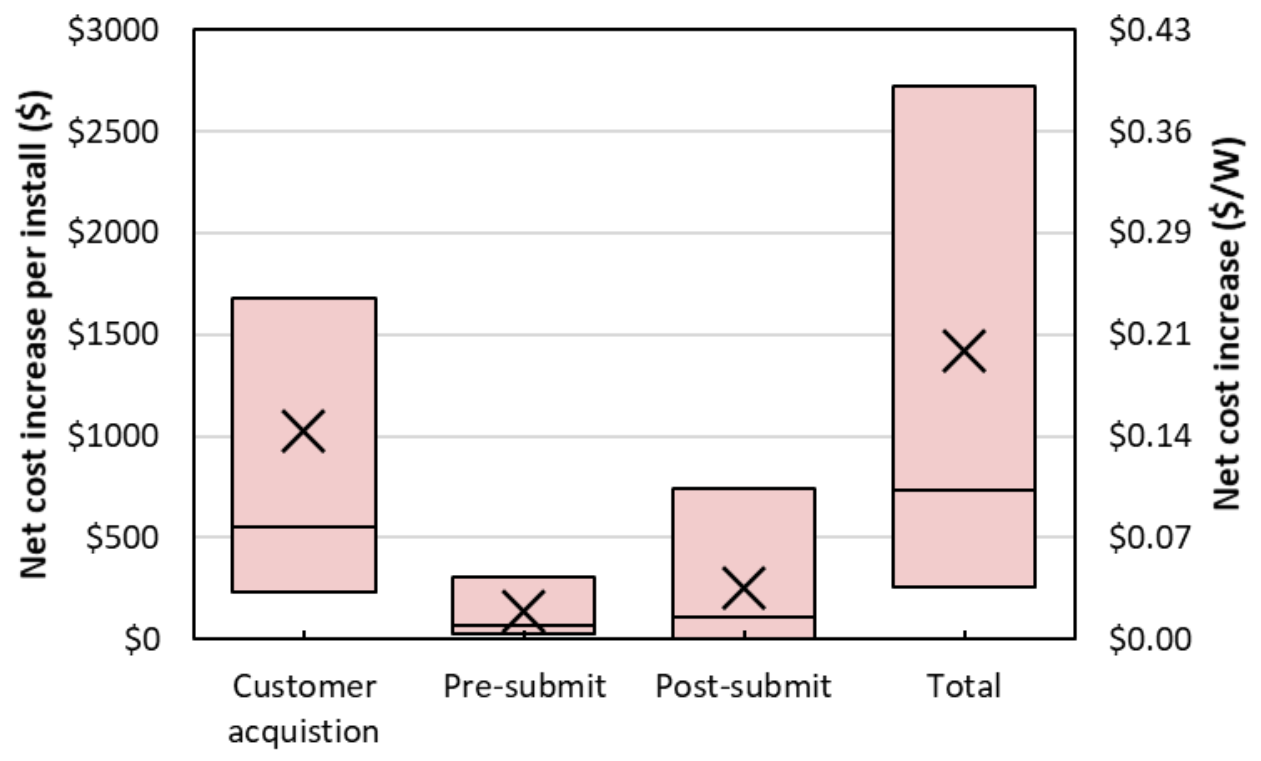

Figure ES-3. Cancellation cost impacts and ranges found by this study, i.e., the increase in installed system soft costs due to contract cancellations. High-low ranges and medians are represented by the boxplots, and averages are denoted by the $X$ 's. Customer acquisition spending on potential customers who never sign contracts is excluded.

In summary, we find that contract cancellations:

- May be more common for medium-to-large installers than previous estimates

- Generally occur earlier in project timelines, though a significant number happen even after permit/ATB approvals

- Do not appear to be driven primarily by delays in permitting and interconnection application reviews

- May contribute significantly to customer acquisition and other soft costs, though the actual impact depends on installer practices. 


\section{Table of Contents}

1 Introduction ...........................................................................................................................

2 The Solar Adoption Process and Contract Cancellations ........................................................ 3

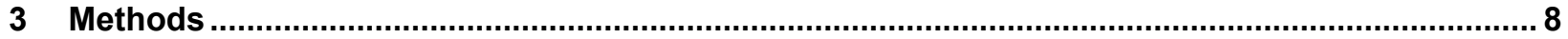

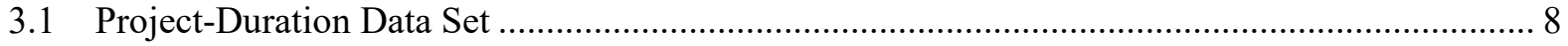

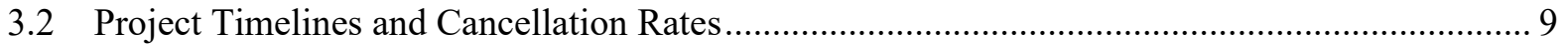

3.3 Cancellation Cost Modeling ............................................................................ 10

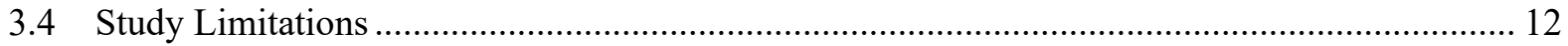

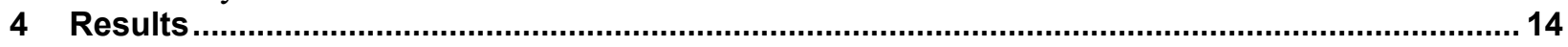

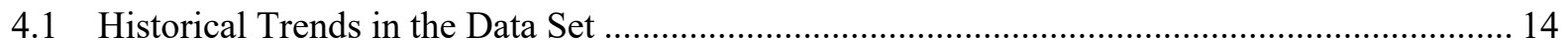

4.2 Cancellation Timelines......................................................................................... 15

4.2.1 Cancellations by Project Phase ............................................................. 15

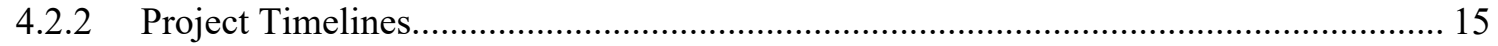

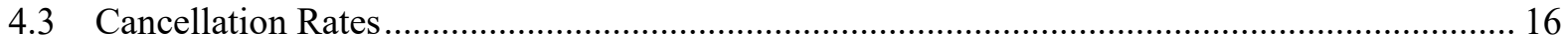

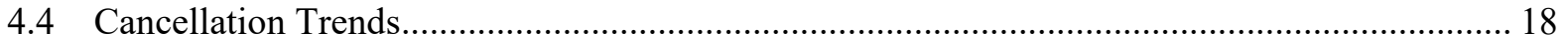

4.4.1 Project Cycle Times .................................................................................. 18

4.4.2 AHJ-Level Trends in Cancellation Rates ............................................................ 18

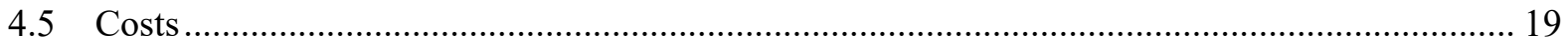

4.5.1 Installer Soft Cost Model ........................................................................... 19

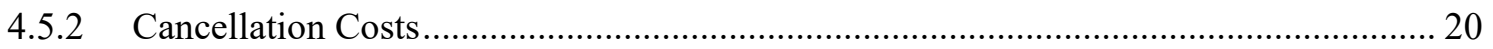

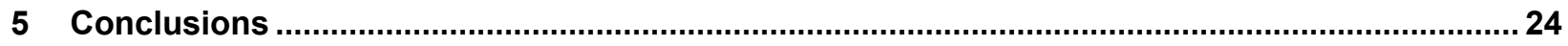

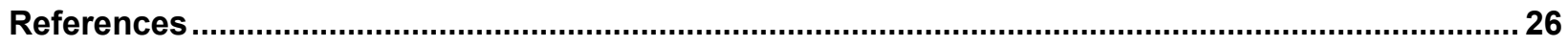

Appendix A. Cancellation Cost Modeling ................................................................................ 28 


\section{List of Figures}

Figure ES-1. Cancellation rates for the post-contract/pre-install phases identified by this study. The average rate is presented for the entire project-level data set, whereas the median and range come from rates across individual installers and years (2017-2019) within the data set. (P/ATB means permit/approval to build application.).....

Figure ES-2. Accumulated installer spending/costs through each phase of the pre-install process. The average and range of costs are based on direct installer spending on a per-project basis, whereas net costs per install include the higher soft costs resulting from spending on unsuccessful projects. Only projects with signed contracts are considered cancellations. Net costs are taken directly from Feldman et al. (2021).

Figure ES-3. Cancellation cost impacts and ranges found by this study, i.e., the increase in installed system soft costs due to contract cancellations. High-low ranges and medians are represented by the boxplots, and averages are denoted by the X's. Customer acquisition spending on potential customers who never sign contracts is excluded...................................................... viii

Figure 1. The general process for residential PV adoption......

Figure 2. Difficulties and concerns cited as reasons that households stopped pursuing PV adoption. Based

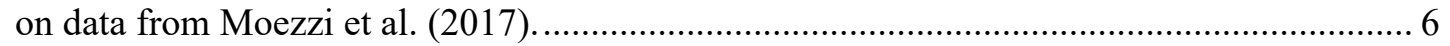

Figure 3. Idle and cancelled projects in the data set by week of last project update............................... 14

Figure 4. Timelines for all unsuccessful (cancelled and idle) projects, from contract signing to last project

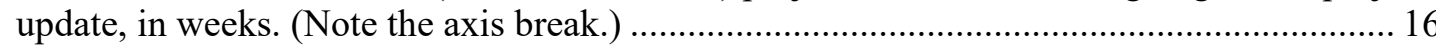

Figure 5. Overall cycle times by project status for the pre-submission and permit/ATB review phases in the data. Averages are marked by an X. Not all outlier data points are shown, but are equally common between reported statuses.

Figure 6. Installer cost accumulation by pre-install phase. Spent costs on a per-project basis are shown in red, with the average spent cost per project indicated by the red line and the modeled range in costs shown as the shaded area. Net cost per installed project is shown as the solid black line. Major phases are delineated by the vertical dotted black lines.

Figure 7. Range in net cost increases for installed projects due to contract cancellations, delineated by spending in each phase. Medians and high-low ranges are shown by the boxplots, with averages marked by an X. Net increases are shown in both cost per installed project $(\$)$ and cost per watt $(\$ / \mathrm{W})$, assuming a $7-\mathrm{kW}$ average system size.

Figure 8. Average accumulation of net soft costs for installed projects by phase and cost type. Noncontract spending is from Feldman et al. (2021) and represents customer acquisition spending on potential customers who never sign contracts.

Figure 9. Cancellation rate model utilized for the results in the report, i.e., using an approximately quadratic decrease in unsuccessful project rates between contract signing and permit submission. 


\section{List of Tables}

Table 1. Project Counts by Status and Year in the Data Set ................................................................. 9

Table 2. Count and Percentage of Total Unsuccessful Projects by Project Phase (Last Completed Step) in the Data Set, Subdivided by Project Status ............................................................................. 15

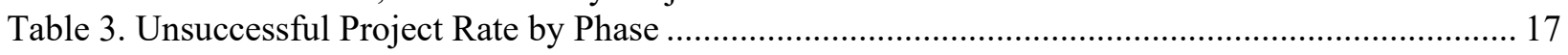

Table 4. Overall Summary of Findings on Post-Contract Cancellation Impacts, Delineated by Project

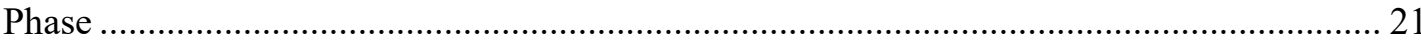

Table 5. Modeled Average Costs and Ranges for Each Phase and Cost Type ...................................... 28

Table 6. Cost and Cancel Rate Scenario Combinations Utilized To Estimate the Cost Increase Due to Unsuccessful Projects, Along With the Unsuccessful Project Rate at Each Major Phase of

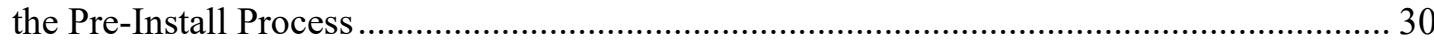

Table 7. Cost Increase Results by Unsuccessful Project Rate Scenario .................................................. 31 


\section{Introduction}

Over 2.8 million households have adopted rooftop solar photovoltaics (PV) in the United States (Davis et al., 2021). Although the number of additional households who signed but later cancelled PV installation contracts is unknown, some estimates suggest it may be 1 in 10 contracts or higher (Cook et al., 2021; Liao, 2020). Some level of PV contract cancellation is likely inevitable, as customers must commit to a financially sizable, long-term investment with uncertain future benefits (Inderst and Ottaviani, 2013). At the same time, installer practices vary regarding deposits, down payments, and/or cancellation penalties, and some or all of these incurred costs may not be recovered by the installer. Unrecovered costs from cancellations increase "soft costs" (non-equipment costs) for installers, and may contribute to higher prices for customers who successfully install PV systems.

Despite the dramatic decline in PV costs over the last decade, soft costs for residential PV in the United States remain high as a percentage of overall install costs (Feldman et al., 2021; O'Shaughnessy et al., 2019; Seel et al., 2014). Several distinct phases in the pre-install timeline contribute to these soft costs, including customer acquisition and the permitting, inspection, and interconnection (PII) process with the local authority having jurisdiction (AHJ) and the utility. The costs associated with contract cancellations are thus dependent on when cancellations occur during the process - all else being equal, installers incur greater costs when customers cancel further into the process. For example, if a customer signs a contract and cancels the following day, the installer will have incurred staff time and other costs for lead generation, sales, and contract drafting/negotiations. However, if a customer signs a contract but does not cancel until several weeks later, the installer will likely incur additional costs, such as system design and preparation, submission, and payment of fees for the permit and interconnection applications. Thus, estimating cost impacts from cancellations requires an accurate assessment of both how many contracts are cancelled in the pre-install phase and when they are cancelled.

Recent studies have examined interrelated topics associated with customer cancellations, including data-derived cancellation rates in California (Liao, 2020); installer-reported rates and drivers of cancellations (Cook et al., 2021); surveyed views, experiences, and concerns from both adopters and non-adopters of rooftop PV (Moezzi et al., 2017); "pain points" in the preinstall process that may lead to cancellations (Sinitskaya et al., 2019); and the impacts of PII timelines, requirements, and delays that may contribute to increased soft costs and are often theorized to be a driver of cancellations (Hsu, 2018; O'Shaughnessy et al., 2020; White, 2019). Altogether, these studies suggest there is no single driver of PV contract cancellations, though customer experiences and finances, the cost/benefit proposition, and the permitting process all likely play a role. A better accounting of when customers cancel in the pre-install timeline may help shed light on their decisions and provide a better guide for future efforts aimed at decreasing the cost impacts of cancellations. 
This study attempts to fill these research gaps through analysis of a project-level data set of residential PV installs and cancellations provided by four medium-to-large-scale installers ${ }^{2}$ from across the United States. The data set included timelines for 98,471 installed and 101,194 unsuccessful residential PV projects of $10 \mathrm{~kW}$ or less between 2017 and 2019. We used the data set to address four main questions: (1) when do cancellations happen in the pre-install project timeline; (2) how often do cancellations happen (i.e., what are cancellation rates at each phase of the process); (3) are there observable trends in cancellations that may point to underlying causes, such as PII delays; and (4) what are the potential cost impacts of cancellations. We find evidence suggesting that contract cancellations may have been previously underestimated and may be a large contributor to residential soft costs.

\footnotetext{
${ }^{2}$ Using the definitions from Cook et al. (2021), medium-scale companies install approximately 100-1,000 systems per year, whereas large-scale companies install more than 1,000. The largest national installers may install more than 10,000 systems per year, but were not tracked as a separate category. Small installers are those that install less than 100 systems per year and make up the majority of companies in the PV installation industry (O'Shaughnessy, 2018).
} 


\section{The Solar Adoption Process and Contract Cancellations}

The residential solar adoption process is summarized in Figure 1. The process can be divided into three main phases: customer acquisition, engineering and permitting, and install/post-install activities.

Phase 1 (customer acquisition) includes the time and costs associated with advertising, lead generation and/or initial contact with the customer, the sales pitch, initial site surveys and system sizing, drafting and negotiating the proposal, and finally, contract signing.

Phase 2 consists of engineering and the PII process. Once the contract is signed, final site surveys, engineering, and system design are completed. If required by the AHJ, additional reviews are also conducted - most commonly structural reviews by a licensed engineer, but occasionally electrical, fire, or zoning reviews as well. The permit application(s) is then completed and submitted to the AHJ, along with any submission or processing fees. If required, a pre-install interconnection/approval to build (ATB) application is also submitted to the utility. ${ }^{3}$ Generally, applications to the AHJ and utility occur concurrently, though approval timelines between them may differ significantly.

After all approvals are received, Phase 3 begins with scheduling and then installing the PV system. The AHJ then conducts all required inspections of the installed PV system. Some utilities may also conduct their own inspections. Inspection results are submitted to the utility as part of the final request for permission to operate (PTO). After review of the application, the utility grants PTO and the PV system is activated.

\footnotetext{
${ }^{3}$ Not all utilities require pre-install approvals for residential PV systems. For example, in California, the three largest investor-owned utilities allow for interconnection applications to be submitted with the final permission to operate (PTO) request. According to SolarTRACE, at least 19 utilities in six states do not require pre-install ATB for residential PV systems (NREL, 2021).
} 


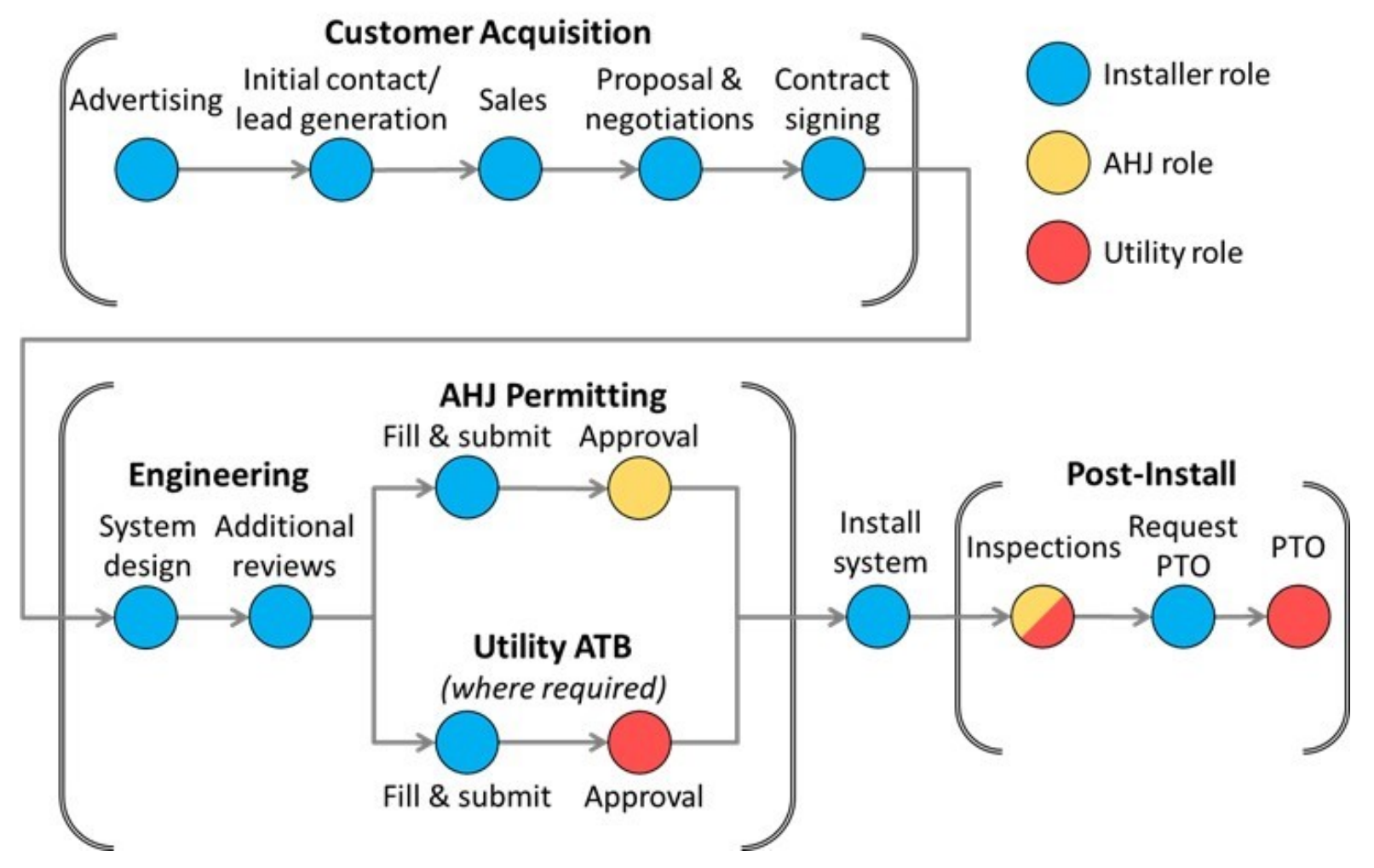

Figure 1. The general process for residential PV adoption.

By the broadest definition, residential PV cancellations encompass any customer with a signed contract who does not install a PV system with that company under that contract. However, it should be noted that what constitutes a "cancellation" differs by company. Nevertheless, "cancellations" uniformly exclude unsuccessful leads in the customer acquisition phase (due to the lack of a signed contract to cancel). Additionally, according to interviewed installers, customers who cancel after install - thus necessitating the removal of the PV system from their homes - are rare. As such, this study will focus primarily on the time between contract signing and system installation, and the term "cancellation" will exclusively refer to unsuccessful projects during this post-contract, pre-install phase. Furthermore, the term "cancellation" will include all unsuccessful contracts, regardless of the decision maker or reason (customer, installer, PII requirements, site limitations, etc.).

Few studies thus far have directly explored residential PV cancellations. Liao (2020) used application data from the California Solar Initiative (CSI) solar rebate program to estimate contract cancellation rates and trends. Using the CSI application as a proxy for contract signing, Liao found that about $12 \%$ of PV contracts were cancelled from 2007-2016. However, although this data set covers about $80 \%$ of California installs prior to 2012, its coverage after that decreases significantly because the program incentive declined. Additionally, one large-scale installer surveyed by Liao found this rate to be lower than their own internal records. Of the cancellations in the data, only $36 \%$ were "officially reported as cancelled" within 1 year of application submission, and the remainder appeared to be "administratively cancelled" after the 1 -year deadline for the rebate program. Official cancellations peak at around 1 month and taper off quickly afterward. Liao found higher cancellation rates for larger systems, for third-party owned systems, and in lower-income areas, with observable cancellation trends affected by 
short-term weather changes (particularly cloudier weather after contract signing). Liao also reported that cancellation policies differ between installers, with some installers imposing no penalties and others charging a $\$ 500-\$ 1,000$ deposit fee (though the data for these findings are anecdotal).

Cook et al. (2021) analyzed a recent survey of 136 small-to-large-scale installers on their experience with project delays and cancellations. Respondents to the survey reported a volumeweighted average cancellation rate of $11 \%$, though half of small installers (less than 100 installs per year) reported no cancellations, while large installers (more than 1,000 installs per year) reported a median cancellation rate of $10 \%$. Permitting delays and changes in customer finances were ranked as the top two reasons for customer cancellations, though mid-to-large installers were much more likely to list customer finances and much less likely to rank PII issues as the primary reasons for cancellation than were smaller installers. Additionally, more than half of respondents $(59 \%)$ reported charging more for installs in areas with more burdensome PII requirements, with a third of those respondents reporting price premiums of $10 \%-20 \%$ or more (i.e., $\$ 0.4-\$ 0.7 / \mathrm{W})$.

Moezzi et al. (2017) surveyed residential PV adopters and non-adopters about their views and experiences with solar. Most relevant were the "considerers," who previously had contact with an installer but ultimately decided against PV adoption. Although this group does not necessarily represent customers who cancelled contracts, their reasons for backing out of the adoption process may elucidate why some customers cancel. The survey data suggest that household finances play a key role in their decisions, with more than half of respondents citing financial difficulties, affordability, taking on debt, investment concerns, and questions about financial returns as difficulties or concerns about PV adoption (Figure 2). Frequently cited non-financial challenges included concerns about equipment and maintenance, impacts to the home and its value, and installer trustworthiness. Notably, PII concerns were cited least by respondents, though it is uncertain whether any of these "considerers" had signed a contract with a solar installer (PII processes typically begin after that step). 


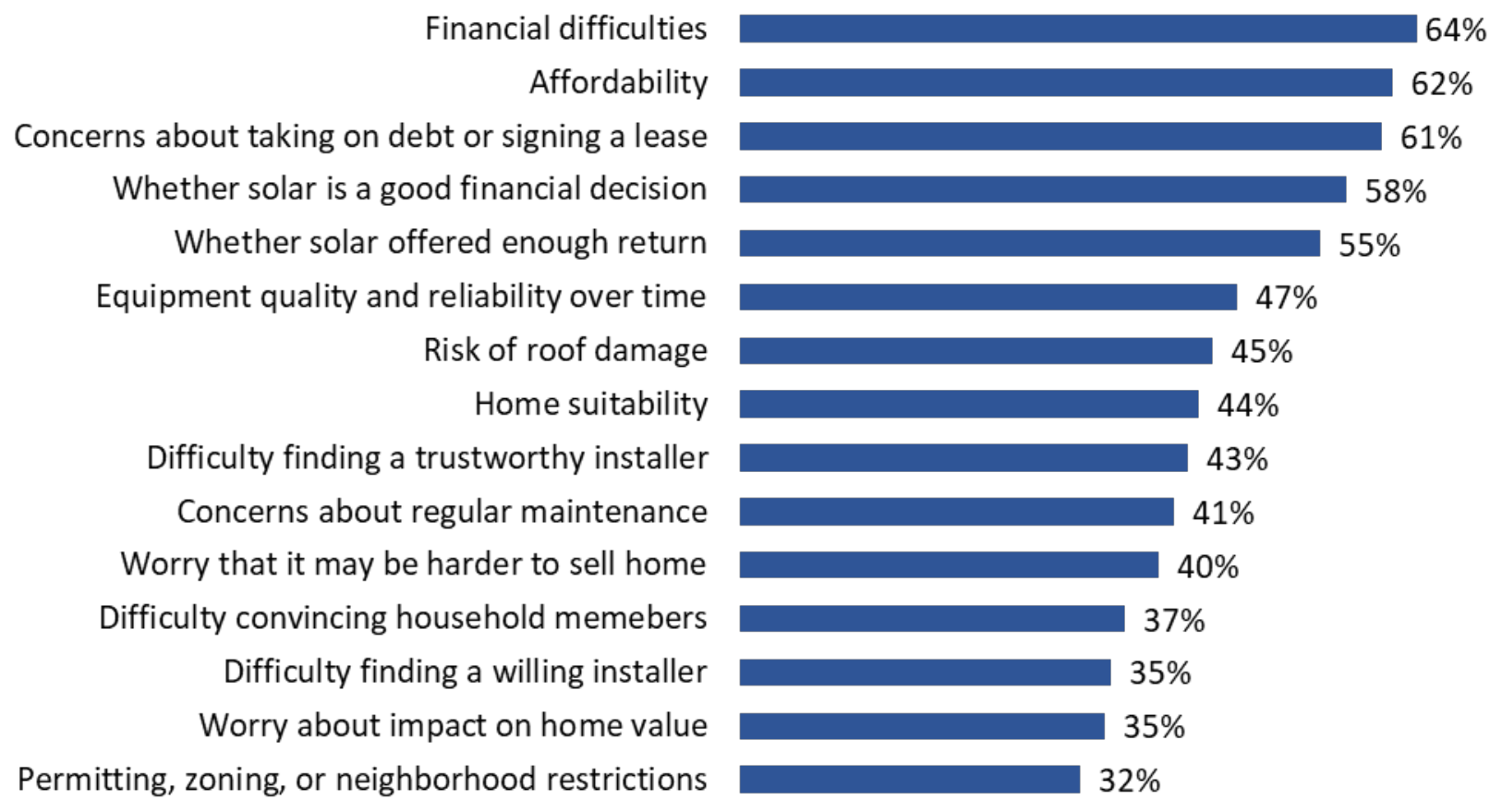

Figure 2. Difficulties and concerns cited as reasons that households stopped pursuing PV adoption. Based on data from Moezzi et al. (2017).

A growing body of literature provides indirect but useful insights into potential PV cancellation drivers and costs. For example, Sinitskaya et al. (2019) identified "pain points" throughout the pre-install process that may be associated with cancellations, such as initial contact between the installer and homeowner, contract negotiations, design changes and roof upgrades (if required), PII processes, and scheduling the install. While the study does not directly discuss cancellations, these pain points may negatively impact the customer experience and thus may contribute to cancellations.

The PII process has seen the most attention in the literature as a driver of project timelines, costs, and potential cancellations. Significant variation in timelines both across and within AHJs exist, and the differences may be attributable to both AHJ policies and installer practices (O’Shaughnessy et al., 2020; Stanfield et al., 2013, 2012). Furthermore, some permitting requirements - especially restrictions from zoning or homeowner associations - are particularly prone to longer approval timelines and project delays. Lengthy durations and delays throughout the PII process are hypothesized to drive contract cancellations, though this correlation has not yet been quantitatively evaluated (O'Shaughnessy et al., 2020). Nevertheless, restrictions on system design parameters (such as size, orientation, or position) may still directly lead to cancellations through impacts on project returns (Cook et al., 2021). Alternatively, improved AHJ permitting processes are likely correlated with higher installation rates, though whether this increase is due to greater sales volume or decreased cancellations has not been evaluated (Hsu, 2018; Parsons and Josefowitz, 2021; White, 2019). Altogether, the research suggests that PII processes impact cancellations, but no study thus far has sought to directly evaluate this impact or its associated costs. 
Benchmarks for soft costs of installed projects provide reference points for estimated cost impacts from cancellations. Although the PII process has again seen the most attention, customer acquisition and marketing costs in the United States are high and are a large contributor to soft costs (Seel et al., 2014). For example, Feldman et al. (2021) modeled customer acquisition costs per successful install at $\$ 0.38-\$ 0.5 / \mathrm{W}$ versus average PII costs of $\$ 0.23-\$ 0.25 / \mathrm{W} .{ }^{4}$ Wood Mackenzie found somewhat higher customer acquisition costs, of $\$ 0.45-\$ 0.65 / \mathrm{W}$ and up to $\$ 0.77 / \mathrm{W}$ or higher for some large installers (Cox, 2020; Mond, 2019). For PII, Burkhardt et al. (2015) found that onerous permitting processes can result in as much as $\$ 0.18 / \mathrm{W}$ of additional installer costs, while Dong and Wiser (2013) estimated permitting cost impacts of $\$ 0.27$ $\$ 0.77 / \mathrm{W}$.

Implicit in some benchmarks are the added costs of unsuccessful projects. Although the average spent cost per potential customer may not be particularly high in the early stages of the adoption process, the average net cost per install is often much higher, because it must incorporate aggregate spending on all potential customers - especially spending lost on customers who do not ultimately complete installs with that company. Because both customer acquisition and PII may account for a significant amount of soft cost accumulation prior to install, distinguishing between spent costs per customer and net costs per install is critical for estimating the cost contribution of contract cancellations.

Only Cook et al. (2021) have attempted to estimate possible cost impacts and/or price premiums due to cancellations. Based on installer feedback, the authors concluded that cancellations may cost $\$ 1,000-\$ 2,000$ per contract, or up to $\$ 5,000$ if cancellations occur during or after permitting and interconnection. Subsequently, the authors modeled several cost scenarios, estimating that cancellations may increase costs by $\$ 0.011-\$ 0.068 / \mathrm{W}$ for larger installers. This range is likely higher if an individual company has a higher cancellation rate than modeled (10\%) and/or does not withhold deposits.

Altogether, the literature shows a gap in broad, data-driven analysis of residential PV cancellations in the United States, particularly in relation to the questions set forth in this paper-namely: when, how often, why, and at what cost?

\footnotetext{
${ }^{4}$ Smaller/more local installers usually report lower marketing and customer acquisition costs than larger installers, in part due to less marketing spending and a greater reliance on customer referrals and other lower-cost sales channels. Thus, their costs are generally modeled to be at the low end of these ranges. On the other hand, larger installers may choose to increase their marketing and customer acquisition spending in order to grow their market share (Feldman et al., 2021; Mond, 2019).
} 


\section{Methods}

To answer these questions, this study leverages data sets and models developed by the National Renewable Energy Laboratory (NREL). First, we evaluate the volume of cancellations and when they occur through an analysis of project-level data from medium-to-large installers in 20172019. Then, we assess the potential cost impacts of these cancellations using NREL's bottom-up cost model (Feldman et al., 2021). Here, we summarize the data collection and methodology at each step in the analysis.

\subsection{Project-Duration Data Set}

This study relies on data collected from medium-to-large installers and published in NREL's Solar Time-Based Residential Analytics and Cycle Time Estimator (SolarTRACE) (NREL, 2021). This data set includes time-stamped dates for customer contract signing; permit submission to and approval by the local AHJ; ATB request submission to and approval by the utility (if applicable); and installation complete. Most projects list the names of the relevant AHJ and utility.

Many installers also provided data for projects that had been cancelled or stalled indefinitely during this period. However, not all installers provided data for projects cancelled during all phases of the pre-install process - some only reported cancellations after a permit/ATB application was submitted. To examine trends in cancellations at all phases of the project between contract signing and install, we developed a sample data set that included only those companies that reported both pre- and post-application cancellations, along with sufficient PIIrelated dates for analysis.

The sample data set consisted of 199,665 projects across four installers that met criteria for use in this study — namely, projects that were (1) standalone PV-only systems; (2) $10 \mathrm{~kW}$ in size or less (i.e., residential projects); and (3) installed, cancelled, or idled (stalled) in 2017-2019. For uninstalled projects, the date of first permit submission or other last project update was used to determine the relevant project year. Altogether, the sample data set covers about $10 \%$ of U.S. installs each year from 2017-2019. ${ }^{5}$ Projects from approximately 2,500 AHJs in 25 states are represented.

A combination of listed dates and company-reported project statuses were used to develop a current (as of mid-2020) status for every listed project, either "installed," "cancelled," "idle," or "in progress." Project counts by year and status are shown in Table 1. Installed projects are those that have completed installation, whether or not they have received final PTO from the utility. Projects were flagged as installed if they listed an install complete date, a PTO granted date, or a company-reported status indicating either. Cancelled projects are those with either a listed cancellation date or an installer-reported status that indicates the project has been closed out and the customer is no longer active with that installer. Idle projects are those that have gone more than 6 months without a project update and have not been installed. ${ }^{6}$ This calculation is

\footnotetext{
${ }^{5}$ Based on quarterly installed project counts from Davis et al. (2021); for comparison, the SolarTRACE dataset covers about $20 \%-30 \%$ of U.S. installs each year from 2017-2019, and additional installer-provided data (excluded from SolarTRACE due to lack of PII information) covers up to 34\% of U.S. installs in 2018.

${ }^{6}$ This timeframe was chosen because less than $1.5 \%$ of successful installs in the data set have a gap between contract and application submission or between application approval and install that is longer than 6 months.
} 
performed separately for each installer based on the last date provided in their data set. However, because many installers in the sample provided data into mid-2020, 99\% of idle projects in this study have actually gone at least 10 months without an update in their respective data sets.

Table 1. Project Counts by Status and Year in the Data Set

Idle projects are those that have gone 6 or more months without a project update. All other statuses are reported by the install company.

\begin{tabular}{lrrrr}
\hline Project Status & $\mathbf{2 0 1 7}$ & $\mathbf{2 0 1 8}$ & $\mathbf{2 0 1 9}$ & Total \\
\hline Installed & 29,211 & 32,705 & 36,555 & 98,471 \\
Cancelled & 12,771 & 15,977 & 16,726 & 45,474 \\
Idle & 7,653 & 17,232 & 30,835 & 55,720 \\
\hline Total Projects & 49,635 & 65,914 & 84,116 & 199,665 \\
Total Unsuccessful & 20,424 & 33,209 & 47,561 & 101,194 \\
\hline
\end{tabular}

As seen in the table, idle projects make up a large and increasing proportion of unsuccessful projects in the data set. Given that what constitutes a "cancellation" differs by company, the actual status and treatment of these idle projects is uncertain. Idle projects could be stalled indefinitely, or could represent unsuccessful or lost leads, actual cancellations, or even potential opportunities to reengage with the customer (possibly under a new contract). However, given the large number of idle projects and their length of time without a project update, we consider them unsuccessful and equivalent to a cancellation of that particular contract.

\subsection{Project Timelines and Cancellation Rates}

This study evaluated project timelines from two perspectives: (1) cancellation timelines, including project dates, phases, and age (since contract signing) at the time of cancellation; and (2) cycle times, or the durations of the completed phases of cancelled projects relative to those of successful installs.

Cancellation timelines identify when projects are cancelled or stall in the post-contract, preinstall process. These timelines are used to calculate cancellation rates across project phases and to show when and how often contracts are cancelled. Based on the available data, we categorized post-contract project phases as follows. The project phase at cancellation is based on the last successfully completed step.

- Pre-PII application submission - begins with the contract signing date and includes system design, engineering, and any additional reviews.

- Application(s) review - begins with the first submission of a permit or ATB application.

- Post-application(s) approval - occurs after the first approval by the AHJ or utility.

- System installation - projects cancelled after installation are very rare and are not considered in this study.

The application review and post-approval phases are combined into a single "post-submission" phase for some sections of this study. 
Project cycle times were calculated for the pre-application and application review phases, and were calculated separately for cancelled, idle, and installed projects. Medians, quartiles, and the $5^{\text {th }}$ and $95^{\text {th }}$ percentiles were calculated to assess whether cancellations were consistently linked with delays (longer timelines) in either phase. Cycle times are averaged across the three years in the data.

Two types of cancellation/unsuccessful project rates were calculated for the data set: an overall rate (delineated by phase) and a by-phase rate. The equations for each are shown below. Average results are presented for the entire data set, but calculations were also performed by year and by installer to assess ranges and medians in the data.

$$
\begin{gathered}
\text { Overall rate }(\%)=\frac{\text { Unsuccessful project count per phase }}{\text { Total project count }} \\
\text { By-phase rate }(\%)=\frac{\text { Unsuccessful project count per phase }}{\text { Active project count at the start of the phase }}
\end{gathered}
$$

In the equations, the unsuccessful project count per phase is the number of projects that do not progress beyond that phase (because they are either cancelled or become idle). The total project count includes all installed and unsuccessful projects in the data set (or all such projects subdivided by year and/or installer). The active project count at the start of the phase is determined by the total project count minus all unsuccessful projects from previous phases.

To validate our results in this study, we compared our calculated cancellation rates with the installer-provided data collected for SolarTRACE-i.e., with cancellation rates from other installers who were excluded from this study. Comparisons included both overall cancellation rates and post-submission-only rates, depending on the data provided by each installer. With these additional installers, the cancellation rate comparisons cover $22 \%-34 \%$ of U.S. residential PV installs from 2017-2019.

We also performed cycle time and cancellation rate calculations at the AHJ level. By doing so, we sought to address two questions. First, are there cycle time differences between successful and unsuccessful projects at the individual AHJ level that may otherwise be masked in the overall results from the entire data set? Second, are there consistent differences between AHJs that warrant further analysis, such as cancellation rates or cycle time differences between successful and unsuccessful projects? Both questions may provide evidence of reasons for contract cancellations.

To ensure sufficient data for comparisons between AHJs, at least 30 projects were required for each cancellation rate and cycle time (successful versus unsuccessful) calculation. AHJs with fewer than 30 projects were excluded from comparisons. In total, cancellation rates from 502 AHJs and cycle times from 167 AHJs were evaluated. Cycle times for installed projects are reported in SolarTRACE (NREL, 2021).

\subsection{Cancellation Cost Modeling}

To better understand installer costs at each pre-install phase of the project, this study leverages the residential PV soft cost models developed by Feldman et al. (2021) for NREL's 2020 U.S. 
Solar Photovoltaic System and Energy Storage Cost Benchmark. This model includes: (1) an itemization of steps in the PII process, with delineated labor and other costs for each; (2) alternative customer acquisition pathways, with the installer cost, success rate, ${ }^{7}$ and proportion of overall projects for each; and (3) overhead and other indirect costs.

Using this soft cost model as a foundation, the current study models the accumulation of installer spent costs per customer through each project phase prior to install. By doing so, this study examines the direct sunk costs to the installer, particularly the difference in costs due to unsuccessful projects in earlier versus later phases in the project timeline. Results are compared to the net cost per successful install reported by Feldman et al. (2021), which averages all costs lost due to cancellations across all projects that were successfully installed. The net cost per install is thus:

$$
N C_{p}=\sum_{p=1}^{P} \frac{C_{S, p}}{1-r_{p}}
$$

where $N C_{p}$ is the net cost per install, $C_{S, p}$ is the spent cost per customer, and $r_{p}$ is the unsuccessful project rate (in phase $p$ for each). $P$ is the total number of phases (up to install). In this expression, $r_{p}$ is the proportion of projects that are active at the start of the phase (and thus accrue costs), but that are ultimately not installed (regardless of whether the project cancels/stalls in this phase or a subsequent phase).

For some project phases, a variety of possible pathways and associated costs exist. As such, this study models both an average cost and a typical low/high range for most costs. A detailed listing of costs and ranges is shown in Table 5 in Appendix A.1. The average cost was taken directly from the soft cost model by removing the scaling from the project success rate. ${ }^{8}$ The low/high ranges were determined by taking worst-case and best-case scenarios for each phase from the soft cost model, such as additional engineering review requirements or labor-intensive submission methods. Ranges for permitting and interconnection fees were not considered in the soft cost model and were instead derived from SolarTRACE (NREL, 2021), though these ranges may not include all AHJs and utilities in the United States.

Indirect costs during the customer acquisition phase are included in both the average costs and low/high ranges, as per the original model. Indirect costs were determined by scaling the total indirect cost for customer acquisition per install $(\$ 1,165$ for sales, marketing, and customer service staff) by the success rate for each step, giving an estimated indirect cost per potential customer. Ancillary upgrade costs outside of the PV system-including to the main breaker

\footnotetext{
${ }^{7}$ Feldman et al. (2021) model average conversion/success rates at each phase of the pre-install process, though they only report the net costs per successfully installed project. This study helps validate their modeling assumptions and breaks out the direct costs of cancellations within their overall results.

${ }^{8}$ Feldman et al. (2021) determined by-phase costs by averaging the incidence rate per cost (i.e., \% of projects requiring a given cost step), the average cost per occurrence, and the overall successful install rate for projects in that phase (i.e., the inverse of the cancellation rate). For example, in the lead generation phase, costs for several different lead types, such as referrals, bidding platforms, channel partners, direct marketing, and door-to-door sales, were averaged by the percentage of projects coming from each lead type, divided by the success rate of each type. This study kept the cost calculation as-is but did not perform the final division by success rate, thus giving an average spent cost per customer.
} 
panel, distribution transformer, or roof - are not considered here. Indirect/overhead costs outside of customer acquisition are similarly excluded, as it is unclear how or whether they would scale for cancellation rate changes greater than a few percent (i.e., whether additional staff would be required, etc.). Soft costs outside of the pre-install phase-including inspection costs, install labor, and company margins - are similarly excluded.

Using these results, this study calculates overall cancellation impacts on residential PV costs on an average per-project and per-watt basis (assuming a 7-kW average system size). These calculations utilize three spent cost per customer scenarios (average, low, and high) and four unsuccessful project rate levels (average, low, high, and median), based on the data from this study. Only unsuccessful contracts are considered - thus, spending on potential leads that never sign a contract is excluded from these cost increases. However, approximate per-project spending on customer acquisition is included for all projects with signed contracts.

The equation for determining the overall cost increase follows a similar format as the total net cost per install equation, with an additional $r$ term in the numerator:

$$
\Delta N C_{P}=\sum_{p=1}^{P} \frac{r_{p}}{1-r_{p}} C_{S, p}
$$

Derivations, details on the unsuccessful project rate assumptions for each cost stage, and additional discussion points are presented in Appendices A.2 and A.3. The cost increase calculations assume that the installer does not require and/or keep a deposit from the potential customer.

\subsection{Study Limitations}

Although this study leverages installer-provided data sets and published cost models, it nevertheless faces limitations in its sources, its analysis, and the applicability of its results. First, this study focuses exclusively on medium-to-large installers, which may skew results and thus not be illustrative of the overall market in the United States - half of residential PV projects and the majority of companies in the United States are smaller installers, which are not represented by this study (O'Shaughnessy, 2018; White, 2021). Smaller installers often report lower cancellation rates than larger installers (Cook et al., 2021), so rates calculated by this study may be higher than the true average for all residential PV contracts in the United States. Similarly, while the soft cost models from Feldman et al. (2021) included separate costs for large versus small installers, only the model for large installers was utilized by this study. The primary difference between the models is that larger installers generally report higher cancellation rates, customer acquisition costs, and net PII costs than smaller installers.

A similar skew toward larger installers may also occur in the data set and results from this study, especially when reporting aggregate and average results. Cancellation rates (both overall and byphase) are particularly sensitive to the rates for the largest installers with the most projects in the data set. Thus, ranges and medians for these calculations are presented where applicable. We do not attempt to ascertain whether the average or median rate is more representative of other medium-to-large installers in the U.S. residential PV market. 
Although we assess the project-level data set for timeline or AHJ trends that may correlate with cancellations, we ultimately do not have direct data on the decision makers or the reasons for cancellation. For example, we do not have data to assess how many cancellations in the pre-PII application submission phase are attributable to the PII process (e.g., restrictions from zoning or homeowner associations) or installer disqualifications (unsuitable site/roof conditions). We likewise do not have project-level data prior to contract signing, and thus cannot evaluate processes or trends in the customer acquisition phase. Finally, data on project activities and dates within the pre-PII application phase were limited, so we could not further analyze when exactly projects were cancelled during this phase or assess the reasons for cancellation.

While many of the "officially cancelled" projects in the data set also include cancellation dates, the accuracy of these dates is uncertain. Similar to the findings of Liao (2020), more than half of the listed cancellation dates occur more than 2 months after the last project update, and more than $10 \%$ occur a year or more after. Given this uncertainty — and in order to include idle and cancelled projects without listed cancellation dates - we use the last project update for analysis and discussion of cancellation timelines, with the caveat that "official cancellation" likely occurs later.

Finally, although we report cancellation costs in terms of impacts to successfully installed systems, we do not have actual data on how installers manage cancellation costs. It may be that some installers instead absorb these costs into their overhead and profit margins, without increasing prices for their customers. Accordingly, the cancellation costs reported here may represent a worst-case scenario, in which all cancellation costs are directly passed on to successful customers. 


\section{Results}

Results on cancellation trends and costs are delineated by project phase and are presented in the following order: historical trends in the study data set; cancellation timelines and ratios by phase; cancellation rates relative to successful projects in each phase; cancellation trends, including cycle time comparisons; and per-project and aggregate costs due to cancellations.

\subsection{Historical Trends in the Data Set}

Historical trends in idle and cancelled projects across the three years in the data set are shown in Figure 3. Totals are shown by week of last project update. The first two months of 2020 are also shown for comparison, though these projects are excluded from analysis. As seen in Figure 3, the number of cancelled projects per week remains relatively constant from 2017-2019, whereas the number of idle projects increases dramatically during this time. Additionally, idle counts increase significantly starting in May 2019 and generally remain high through February 2020. This trend suggests that overall cancellation rates may have been increasing during this time, though the cause is unknown. ${ }^{9}$ (Dips in the figure correspond to Thanksgiving and the winter holidays, when fewer project updates occur due to customer travel and $\mathrm{AHJ} /$ other office closures.)

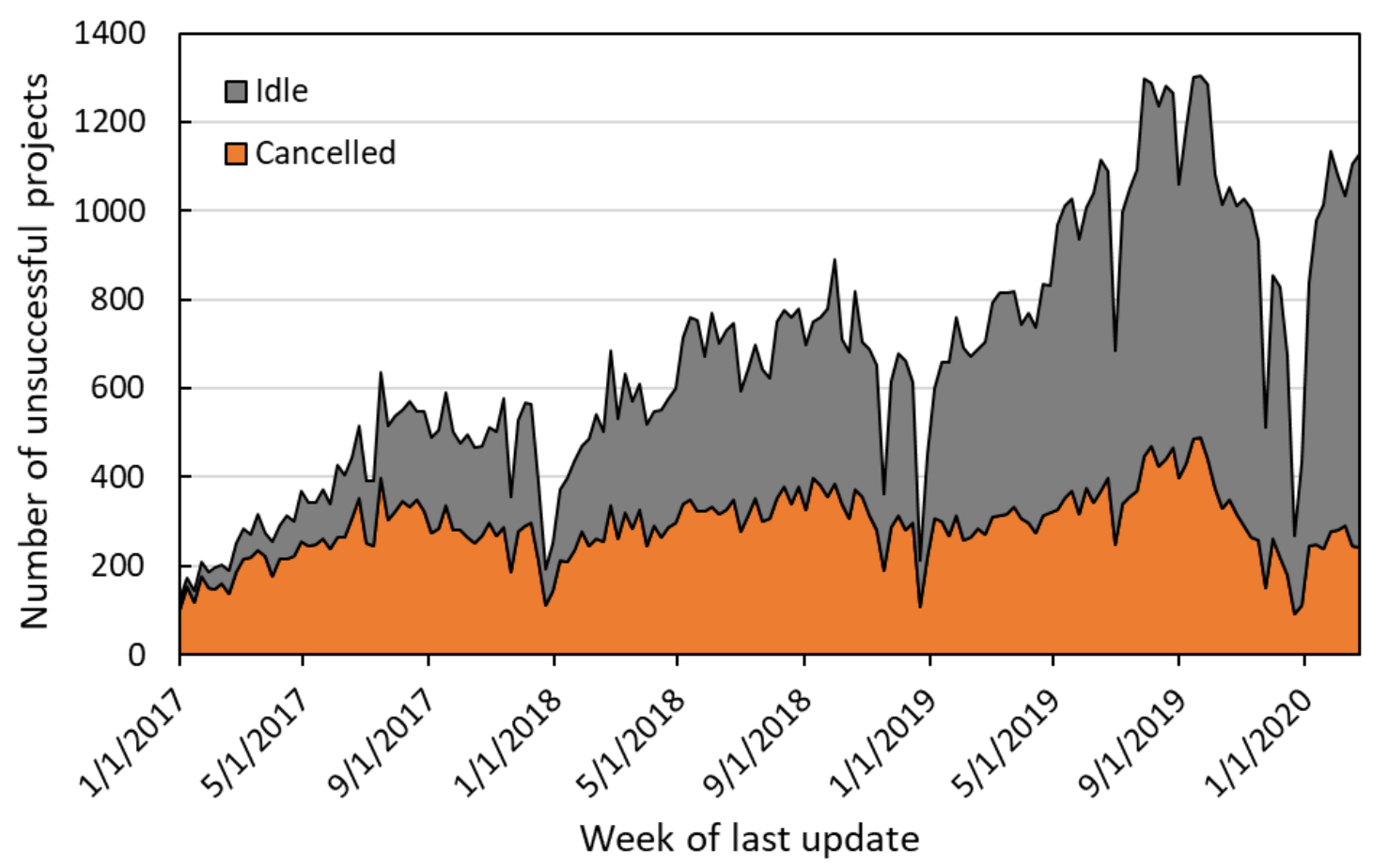

Figure 3. Idle and cancelled projects in the data set by week of last project update.

${ }^{9}$ At the other end of the timeline, the low idle counts through mid-2017 may be an artifact of the data sets provided by installers, though again, the exact cause could not be determined. 


\subsection{Cancellation Timelines}

\subsubsection{Cancellations by Project Phase}

The count and percentage of all unsuccessful contracts in the data set in each project phase are shown in Table 2. Results are also delineated by project status (cancelled versus idle). The range shows the variation between each year in the data set as well as between the 3-year averages of each installer. As shown in the table, the majority (73\%) of unsuccessful projects stall in the presubmission phase. Most of the remainder (25\%) stall after at least one approved application, with only a minor fraction (2\%) stalling during application review. Though there is some variation in these ratios across years and installers, the overall trend remains the same - two-thirds to threequarters of unsuccessful projects stall prior to permit/ATB submission, and most of the rest stall between application approval and install.

Table 2. Count and Percentage of Total Unsuccessful Projects by Project Phase (Last Completed Step) in the Data Set, Subdivided by Project Status

The range shows the variation in by-phase percentage of total unsuccessful projects between each of the 3 years (2017-2019) in the data set and between each installer average across the years.

\begin{tabular}{crrrrrrr}
\hline & \multicolumn{2}{c}{ Cancelled } & \multicolumn{2}{c}{ Idle } & \multicolumn{3}{c}{ Total Unsuccessful } \\
Project Phase & N & \% & N & \% & N & $\%$ & Range \\
\hline Pre-Submit & 27,387 & $60 \%$ & 46,968 & $84 \%$ & $\mathbf{7 4 , 3 5 5}$ & $\mathbf{7 3 \%}$ & $\mathbf{6 8 \% - 7 7 \%}$ \\
\hline Application Review & 1,397 & $3.1 \%$ & 613 & $1.1 \%$ & $\mathbf{2 , 0 1 0}$ & $\mathbf{2 . 0 \%}$ & $\mathbf{1 . 8 \% - 8 \%}$ \\
Post-Approval & 16,690 & $37 \%$ & 8,139 & $15 \%$ & $\mathbf{2 4 , 8 2 9}$ & $\mathbf{2 5 \%}$ & $\mathbf{1 8 \% - 3 0 \%}$ \\
\hline Total & $\mathbf{4 5 , 4 7 4}$ & & $\mathbf{5 5 , 7 2 0}$ & & $\mathbf{1 0 1 , 1 9 4}$ & $\mathbf{1 0 0 \%}$ & \\
\hline
\end{tabular}

The difference in these ratios for projects that are "officially cancelled" versus those that become idle may illustrate the terminology used by installers in tracking unsuccessful projects. Projects that reach the approval phase before stalling are much more likely to be officially cancelled rather than simply remaining idle. Conversely, idle projects are most heavily skewed toward those that stall early on (in the pre-submit phase). Several possible explanations may exist-for instance, installers invest more time and cost in projects that go through permitting and thus track those projects more carefully, whereas idle projects are lower-cost and may still provide future opportunities for reengagement. Additionally, once permitting and ATB are completed, the company looks to schedule the installation, thus presenting the customer with their final go/nogo decision point for either proceeding with install or choosing to cancel the contract.

\subsubsection{Project Timelines}

Project timelines for all unsuccessful projects, from contract signing to last project update, are shown in Figure 4, subdivided by last completed project phase. Again, the figure shows only when the project stalled, not when it was officially cancelled (if it was). As seen in the figure, the majority (72\%) of projects stall in the first week (within 5 business days). Most of these projects list only a contract date and no others, though dates for subsequent work in this phase (such as site surveys and engineering design) are not well-represented in the data. Thus, actual timelines 
before stalling may be somewhat longer than represented. Nevertheless, post-approval projects make up the majority of unsuccessful projects in the subsequent weeks, with a peak 2 weeks after contract signing, followed by a gradual taper. In all, $90 \%$ of projects have a final update within 5 weeks of contract signing.

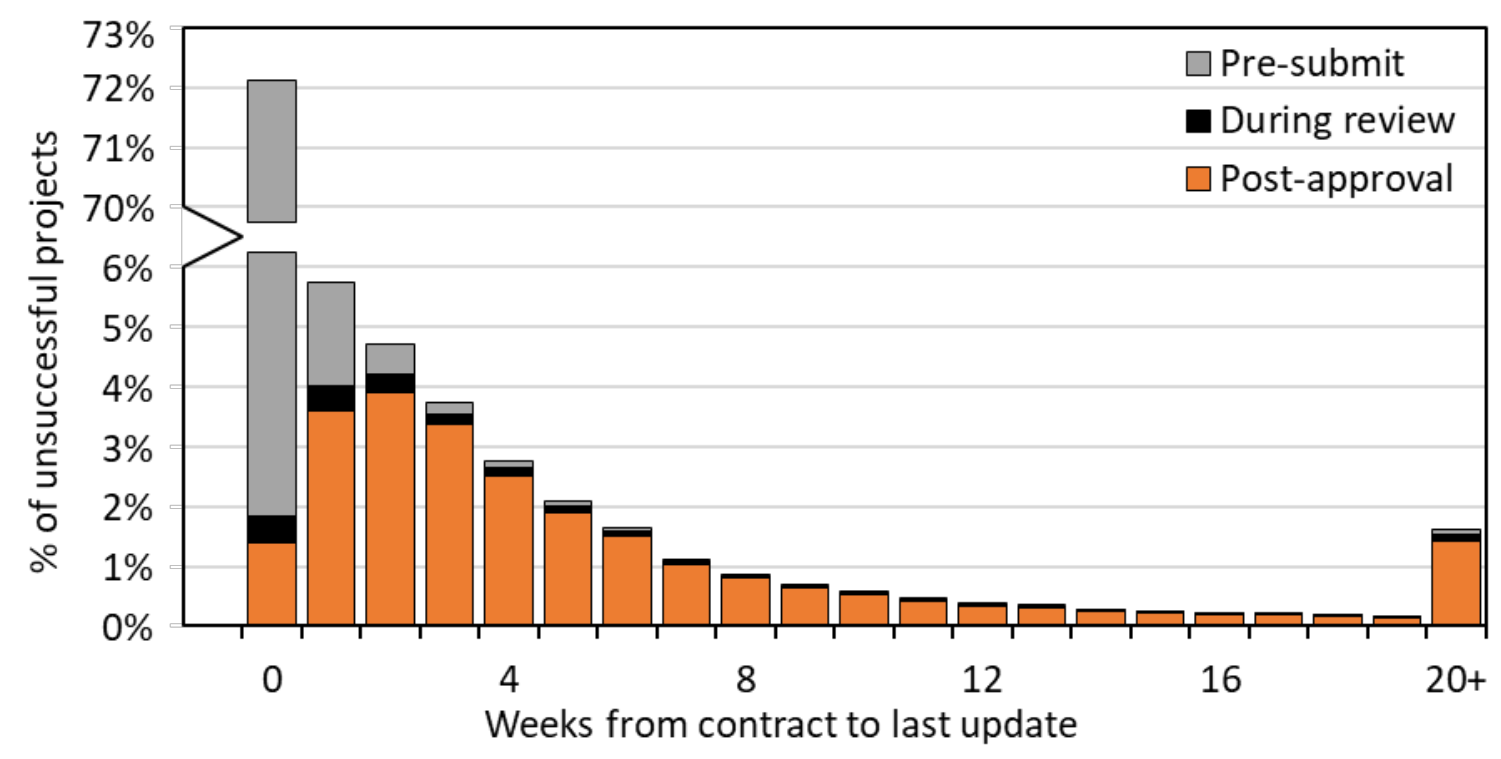

Figure 4. Timelines for all unsuccessful (cancelled and idle) projects, from contract signing to last project update, in weeks. (Note the axis break.)

\subsection{Cancellation Rates}

The rate of unsuccessful projects relative to successful installs is shown in Table 3; again, the rates are delineated by project phase. Two rates are presented for each phase: an overall rate (relative to the total number of both installed and unsuccessful projects), and a by-phase rate (relative only to projects that were active at the start of that phase, excluding unsuccessful projects from earlier phases). For example, as shown in the table, $13 \%$ of all projects in the data set are cancelled or stall in the post-approval phase (left), whereas $20 \%$ of projects that receive permit/ATB approval will still ultimately be cancelled. 
Table 3. Unsuccessful Project Rate by Phase

(Left) Rates in relation to all projects in the data set (installed and total unsuccessful), and (right) rates in relation to only projects active at the start of each phase (excluding unsuccessful projects from earlier phases). The ranges show the rates between installers in the data set, with each company's overall cancellation rate as the primary driver of differences.

\begin{tabular}{ccccc}
\hline Project Phase & $\begin{array}{c}\text { Overall Rate } \\
\text { (\% of all projects) }\end{array}$ & $\begin{array}{c}\text { Range } \\
\text { (Median) }\end{array}$ & $\begin{array}{c}\text { By-Phase Rate } \\
\text { (\% active in phase) }\end{array}$ & $\begin{array}{c}\text { Range } \\
\text { (Median) }\end{array}$ \\
\hline Pre-Submit & $37 \%$ & $\begin{array}{c}16 \%-41 \% \\
(24 \%)\end{array}$ & $37 \%$ & $\begin{array}{c}16 \%-41 \% \\
(24 \%)\end{array}$ \\
Application Review & $1.0 \%$ & $\begin{array}{c}0.7 \%-2.6 \% \\
(1.9 \%)\end{array}$ & $1.6 \%$ & $\begin{array}{c}0.9 \%-3.3 \% \\
(1.4 \%)\end{array}$ \\
Post-Approval & $13 \%$ & $\begin{array}{c}5 \%-14 \% \\
(7 \%)\end{array}$ & $20 \%$ & $\begin{array}{c}6 \%-24 \% \\
(9 \%)\end{array}$ \\
\hline $\begin{array}{c}\text { Overall Data Set } \\
\text { Post-Submit } \\
\text { Only }\end{array}$ & $51 \%$ & $\begin{array}{c}23 \%-55 \% \\
(33 \%)\end{array}$ & - & - \\
\hline
\end{tabular}

*Only includes projects that submitted at least one permit or ATB application-i.e., excludes pre-submit cancellations.

In this medium-to-large installer data set, the overall rate of unsuccessful projects is $51 \%$-i.e., half of all customers in the data set do not ultimately install PV systems under that particular contract. Even when projects that never submitted a permit/ATB application are excluded, the unsuccessful project rate is still $22 \%$ (bottom row), implying that almost a quarter of projects that start the PII process fail to install PV systems. However, these average rates belie significant differences between installers, with the median and lower range rates generally much smaller than the overall averages. For example, the median unsuccessful project rate is $33 \%$, and individual installers have a rate as low as 23\%. (For post-submission projects only, these numbers are $11 \%$ and $8 \%$, respectively.) Although the larger installers in the study data set clearly drive the higher average rates, it is not clear whether the median or average rate is more accurate for other medium-to-large installers in the United States.

These cancellation rates were partially compared against the other installer data collected for SolarTRACE (covering 22\%-34\% of U.S. residential installs). Rates from these other installers generally align with this study; that is, overall rates as high as $40 \%-50 \%$, and post-submissiononly rates of $5 \%-15 \%$. Additionally, the fact that some installers only reported projects cancelled after permit/ATB submission may suggest (albeit circumstantially) that unsuccessful projects from the pre-application phase are not always seen as cancellations. Nevertheless, these findings suggest that focusing only on later-phase cancellations hides the true incidence of unsuccessful contracts, especially given the much higher rates in the pre-application phase.

Even with the ranges seen in the results, the unsuccessful project rates in this study are significantly higher than the $11 \%-12 \%$ rates previously reported (Cook et al., 2021; Liao, 2020). However, the post-submission rate and range are much more closely aligned, suggesting possible differences in terminology and methodology between this and the previous studies. For example, medium-to-large installers may have only reported post-submission cancellations in their 
responses to the survey in Cook et al. (2021), and may not even consider unsuccessful presubmission projects as "official cancellations." Likewise, Liao (2020) only utilizes data for projects for which an incentive application was submitted, so earlier-phase cancellations would not appear in their data at all.

\subsection{Cancellation Trends}

This study evaluated two hypothesized drivers of higher cancellation rates: lengthy project cycle times and AHJ-level differences. Neither was found to be specifically associated with cancelled projects or higher cancellation rates, at least in this data set.

\subsubsection{Project Cycle Times}

Cycle times for the pre-submission and application review phases of all projects in the data set are shown in Figure 5, subdivided by project status (cancelled, idle, or installed). As can be seen, there are no significant differences between cycle times for installed projects and those that are cancelled or idle. Differences between cycle times for each status type are within 1-2 business days at the quartiles, medians, and $5^{\text {th }}$ percentiles, and are generally within a week of each other at the $95^{\text {th }}$ percentile. Although these findings do not preclude cancellation impacts from extended cycle times and/or PII delays, they do suggest that longer cycle times are not specifically associated with unsuccessful projects.
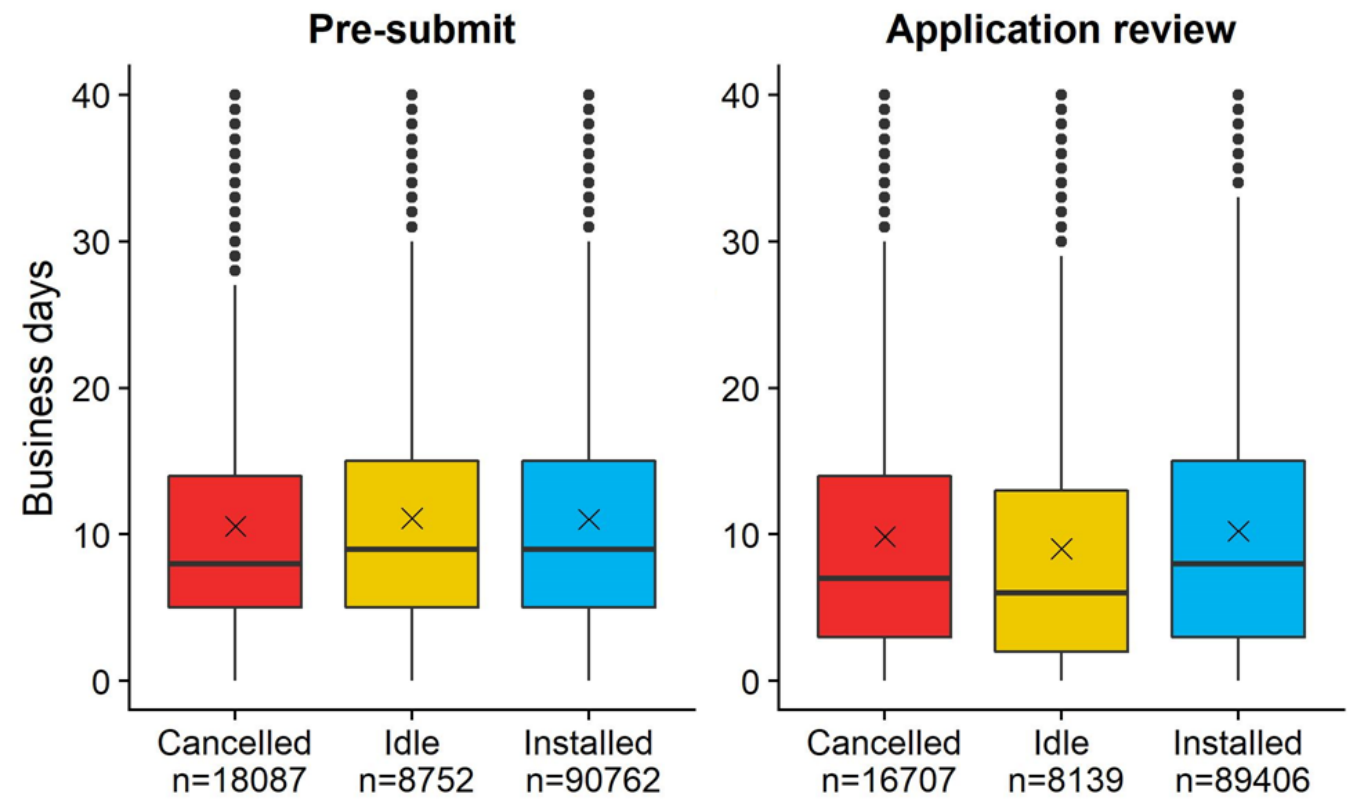

Figure 5. Overall cycle times by project status for the pre-submission and permit/ATB review phases in the data. Averages are marked by an X. Not all outlier data points are shown, but are equally common between reported statuses.

\subsubsection{AHJ-Level Trends in Cancellation Rates}

Significant differences in both overall and post-submission cancellation rates were found across AHJs in the data set. However, these differences appear to be driven by differences between installers in the data set. For example, AHJs with significantly lower cancellation rates are often served primarily by installers with lower company-wide cancellation rates. For AHJs served by 
multiple installers, the relative ranking of AHJs with lower versus higher cancellation rates similarly varies by installer-AHJs with lower cancellations rates reported by one installer are often not correlated with lower rates reported by another installer. Additionally, no AHJ in the data set is served by more than two installers, so cross-referencing across installers is not possible. Altogether, given these challenges, the data available in this study was insufficient to assess cancellation rate differences between AHJs.

Cycle time differences between installed and cancelled projects were also evaluated at the AHJ level. However, no significant differences were found in application review timelines for installed versus cancelled projects: at the median cycle time for each project status, $90 \%$ of AHJs had approval timelines that were within 2 days of each other, whereas $96 \%$ of AHJs had timelines within a week of each other. These counts decrease only slightly at the third quartile of cycle times, to $74 \%$ and $89 \%$ of AHJs, respectively. These trends, along with the finding in Table 3 that less than $2 \%$ of projects cancel or stall during the application review process, suggest that most cancelled contracts in most AHJs are not associated with longer permit approval timelines than are installed projects.

Of course, it remains possible that some projects in some AHJs (and utilities) may face protracted review and approval timelines that may contribute to their cancellations. Difficulties from other PII requirements (such as zoning, fire, or homeowner association restrictions) may also contribute to cancellations, and at least one interviewed installer claimed that half of their cancellations are ultimately attributable to the PII process. However, the lack of data on the reasons for cancellation and the lack of strong trends by AHJ means that these challenges could not be further evaluated by this study. Additional research is necessary to better examine how PII processes may impact contract cancellations.

\subsection{Costs}

\subsubsection{Installer Soft Cost Model}

Regardless of the cause, cancellations result in real costs to installers, as modeled in Figure 6. The average spent cost per project is shown as the red line, with the modeled high-low cost range shown as the shaded area. Also shown is the net cost per successful install (black line), as modeled by Feldman et al. (2021). ${ }^{10}$ The major pre-install phases - contract signing and permit/ATB submission - are delineated by vertical dotted black lines. As can be seen, installer spending on customer acquisition averages about $\$ 1,000$ at contract signing. Final system design does not add much to installer spending, so contract cancellations earlier in the pre-submission phase average about $\$ 1,000-\$ 1,500$ per project. However, once the PII process begins (starting at "additional reviews" in the figure), costs may escalate significantly, especially at the higher end of the range: the average cost per project increases to over $\$ 2,000$ by permit/ATB approval, but may range as high as $\$ 4,000$ or more for worst-case scenarios (i.e., high-cost AHJ permit and/or utility ATB application requirements).

\footnotetext{
${ }^{10}$ Although moderate differences in net cost per successful install between small- and large-scale installers are reported by Feldman et al. (2021), the differences in cost per project are small enough that we do not present them separately in this study, especially given the large range in costs.
} 


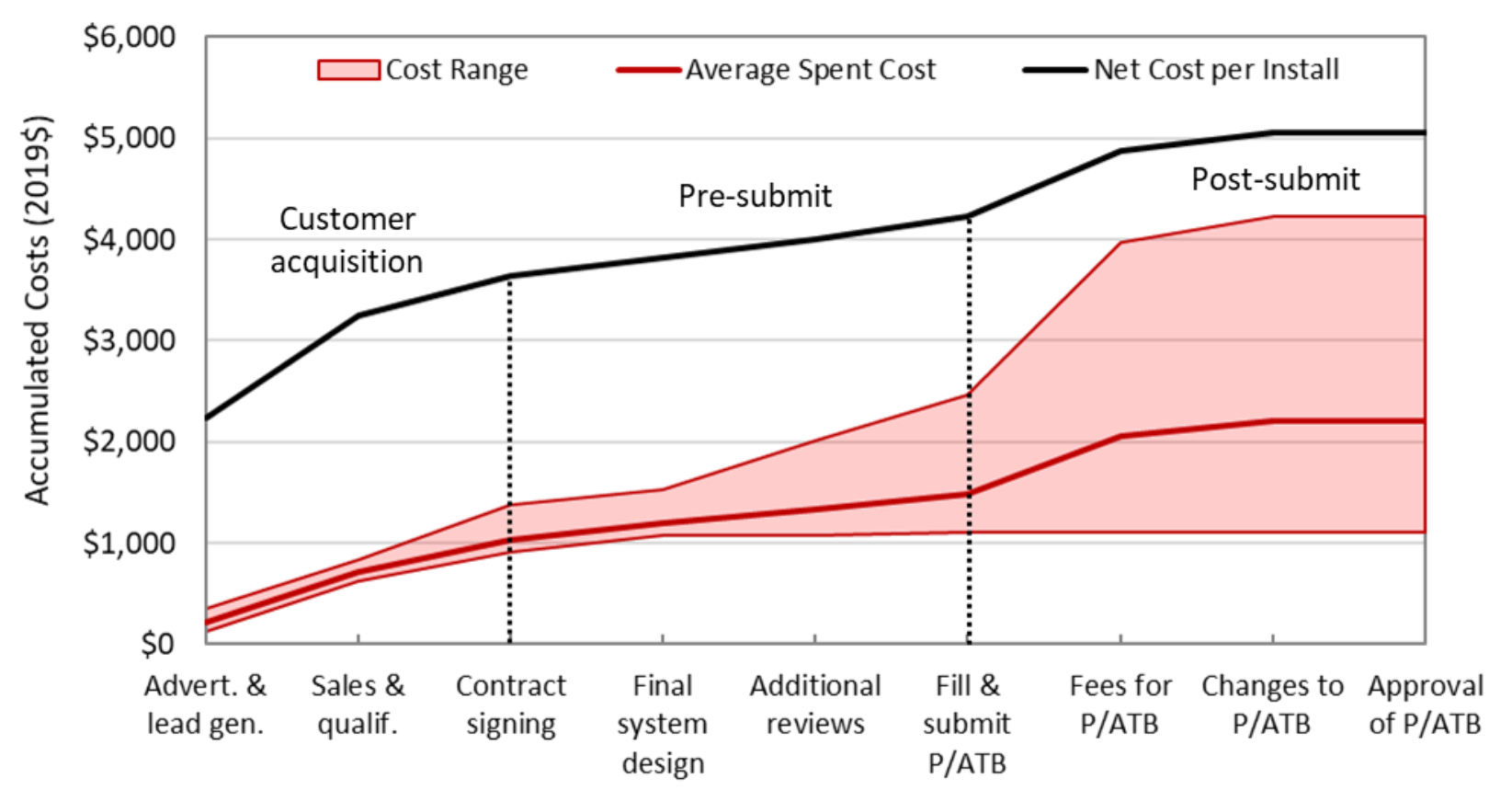

Figure 6. Installer cost accumulation by pre-install phase. Spent costs on a per-project basis are shown in red, with the average spent cost per project indicated by the red line and the modeled range in costs shown as the shaded area. Net cost per installed project is shown as the solid black line. Major phases are delineated by the vertical dotted black lines.

As seen in Figure 6, net costs per successful install are significantly higher than the average spent costs per project, with a $\$ 2,500$ difference at contract signing and an almost $\$ 3,000$ difference by permit/ATB approval. This difference is driven primarily by the relatively low project conversion (success) rates during customer acquisition. While this study did not consider unsuccessful projects prior to contract signing, Feldman et al. (2021) model only about a 10\%$15 \%$ project success rate during the advertising and lead generation stages for medium-to-large installers. ${ }^{11}$ Thus, although spending on advertising and lead generation is relatively small on a per-potential-customer basis, successful projects must incorporate the lost spending on the $85 \%$ $90 \%$ of potential customers who ultimately do not complete installs.

The range in PII costs in Figure 6 shows the impact of AHJ permit and/or utility ATB application requirements on overall soft costs, particularly in higher-cost jurisdictions. Higher cost requirements may include whether and what kinds of professional engineering reviews are required ("additional reviews"); application submission costs (with in-person submissions, especially those requiring multiple trips, as the most expensive); and application fees (modeled scenarios range from $\$ 0-\$ 1,500$ in AHJ and utility fees). Additionally, production limitations, required setbacks, or zoning/homeowner association codes may limit PV system sizes and further increase overall costs.

\subsubsection{Cancellation Costs}

Based on the findings from this study, the overall estimated cost increase to installed systems due to contract cancellations is shown in Figure 7 and Table 4. Again, only post-contract

\footnotetext{
${ }^{11}$ Small installers are modeled by Feldman et al. (2021) with lower customer acquisition spending and higher conversion rates throughout the pre-install process.
} 
cancellations are considered-customer acquisition spending on potential customers who never signed contracts is excluded from the cost increases shown. However, customer acquisition spending on projects that did sign contracts is included.

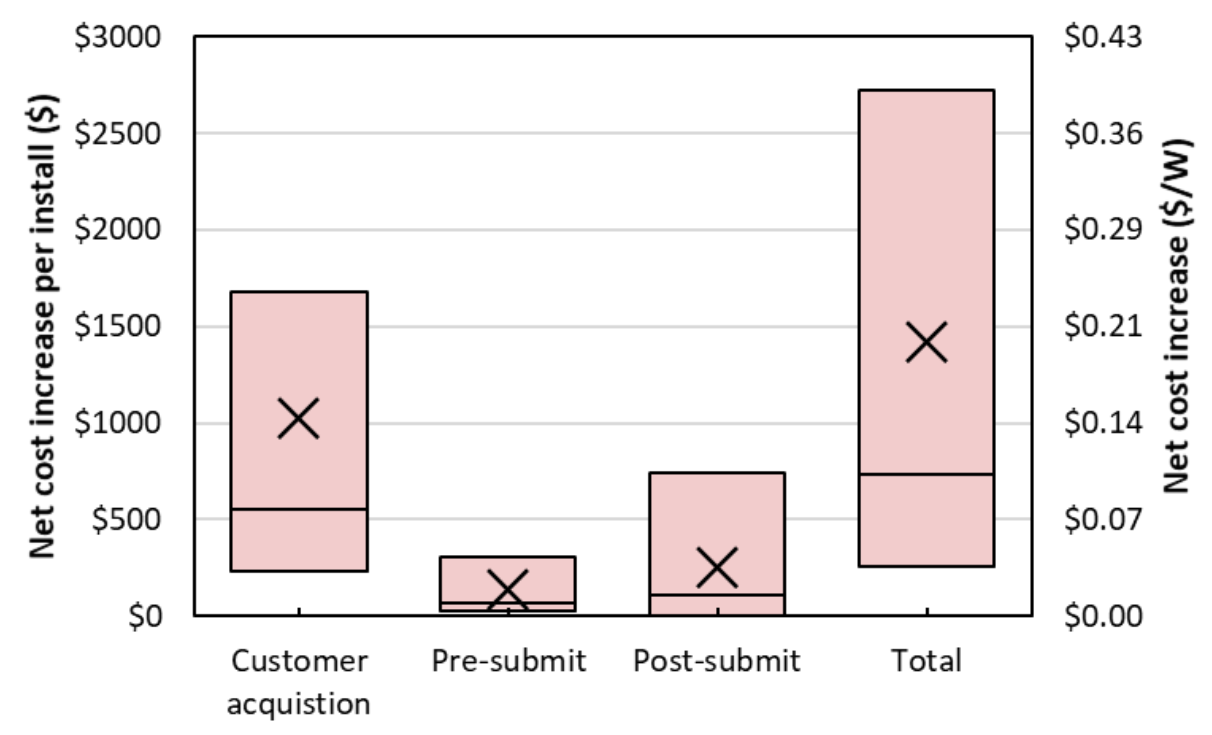

Figure 7. Range in net cost increases for installed projects due to contract cancellations, delineated by spending in each phase. Medians and high-low ranges are shown by the boxplots, with averages marked by an $X$. Net increases are shown in both cost per installed project (\$) and cost per watt $(\$ / W)$, assuming a $7-k W$ average system size.

Table 4. Overall Summary of Findings on Post-Contract Cancellation Impacts, Delineated by Project Phase

Values shown include cancellation rate, accumulated spending lost per cancellation in each phase, and resulting increase in net costs for installed projects (per project and per watt, assuming 7-kW average system size).

\begin{tabular}{|c|c|c|c|c|}
\hline Phase & $\begin{array}{c}\text { Contract } \\
\text { Cancellation } \\
\text { Rate } \\
\text { (\% of installs) }\end{array}$ & $\begin{array}{l}\text { Accumulated } \\
\text { Spent Costs } \\
\text { per Contract }\end{array}$ & $\begin{array}{c}\text { Net \$ per Install: } \\
\text { Average Range } \\
\text { (Median) }\end{array}$ & $\begin{array}{c}\text { Net \$/W: } \\
\text { Average Range } \\
\text { (Median) }\end{array}$ \\
\hline $\begin{array}{l}\text { Customer } \\
\text { Acquisition* }\end{array}$ & $N A^{*}$ & $\$ 1,000-\$ 1,300$ & $\begin{array}{c}\$ 1,030 * \\
\$ 230-\$ 1,680 \\
(\$ 550)\end{array}$ & $\begin{array}{c}\$ 0.147^{*} \\
\$ 0.033-\$ 0.240 \\
(\$ 0.079)\end{array}$ \\
\hline $\begin{array}{c}\text { Pre-Submit } \\
\text { (Post- } \\
\text { Contract) }\end{array}$ & $15 \%-40 \%$ & $\$ 1,000-\$ 2,000$ & $\begin{array}{c}\$ 140 \\
\$ 30-\$ 300 \\
(\$ 70)\end{array}$ & $\begin{array}{c}\$ 0.020 \\
\$ 0.004-\$ 0.043 \\
(\$ 0.010)\end{array}$ \\
\hline Post-Submit & $8 \%-20 \%$ & $\$ 1,000-\$ 4,000+$ & $\begin{array}{c}\$ 250 \\
\$ 3-\$ 740 \\
(\$ 110)\end{array}$ & $\begin{array}{c}\$ 0.036 \\
\$ 0-\$ 0.106 \\
(\$ 0.016)\end{array}$ \\
\hline $\begin{array}{l}\text { Total } \\
\text { (Post- } \\
\text { Contract) }\end{array}$ & $20 \%-50 \%+$ & & $\begin{array}{c}\$ 1420 \\
\$ 260-\$ 2,720 \\
(\$ 730)\end{array}$ & $\begin{array}{c}\$ 0.202 \\
\$ 0.037-\$ 0.388 \\
(\$ 0.105)\end{array}$ \\
\hline
\end{tabular}

${ }^{*}$ Excludes unsuccessful projects prior to contract-i.e., cost increase is only from post-contract cancellations. If all potential customers during the customer acquisition phase are included, the net cost increase per install for this phase increases to $\$ 2,500 \mid \$ 0.36 / \mathrm{W}$, while the total net cost increases to an average of $\$ 3,240 \mid \$ 0.46 / \mathrm{W}$. 
Altogether, contract cancellations were found to add an average of $\$ 0.20 / \mathrm{W}$ to installed system costs, or about $\$ 1,400$ per project. However, there is a significant range in the results, with total increases ranging from $\$ 0.04 / \mathrm{W}$ ( $\$ 260$ per project) to $\$ 0.40 / \mathrm{W}(\$ 2,700$ per project) or higher. The median is about half of the average, at $\$ 0.10 / \mathrm{W}$ ( $\$ 700$ per project). These results are much higher than the estimate range reported by Cook et al. (2021) of $\$ 0.011-\$ 0.068 / \mathrm{W}$, a difference explained by the more detailed cost modeling and higher cancellation rates in this study.

Despite excluding spending on potential customers who never sign a contract, customer acquisition spending on post-contract cancellations still makes up the largest contributor to the net cost increase - almost $75 \%$ of the average cost increase, or $\$ 0.15 / \mathrm{W}$. The impact from customer acquisition spending is four times higher than the impact from permitting and interconnection. This result is not surprising; although cancellations during permit/ATB review or post-approval are twice as expensive than earlier cancellations, they are also much rarer (i.e., only $20 \%-30 \%$ of all cancellations). As such, both the high per-project cost for customer acquisition and the high rate of early contract cancellations appear to be the primary drivers of net cost increases for installed projects.

Figure 8 shows the accumulation of total direct soft $\operatorname{costs}^{12}$ by pre-install phase, delineated by direct per-project spending; cost increases from contract cancellations; and lost spending on noncontract leads during customer acquisition (from Feldman et al. (2021)). As seen, pre-install soft costs average about $\$ 0.75 / \mathrm{W}(\$ 5,200)$ per install, of which about $\$ 0.50 / \mathrm{W}(\$ 3,500)$ come from customer acquisition. Most importantly, net costs from spending on non-contract leads during the customer acquisition phase are 1.5 times higher than costs incurred from contract cancellations: $\$ 0.21 / \mathrm{W}(\$ 1,500)$ versus $\$ 0.14 / \mathrm{W}(\$ 1,000)$, respectively. In addition, contract cancellation costs in the pre- and post-submit phases are relatively small compared to both direct spending in those phases and especially to lost customer acquisition spending (with and without signed contracts).

\footnotetext{
${ }^{12}$ Excluding overhead, install labor, taxes, installer margins, and other indirect costs, which altogether bring soft
} costs up to about $\$ 1.5 / \mathrm{W}$ (around $\$ 10,500$ per project) (Feldman et al., 2021). 


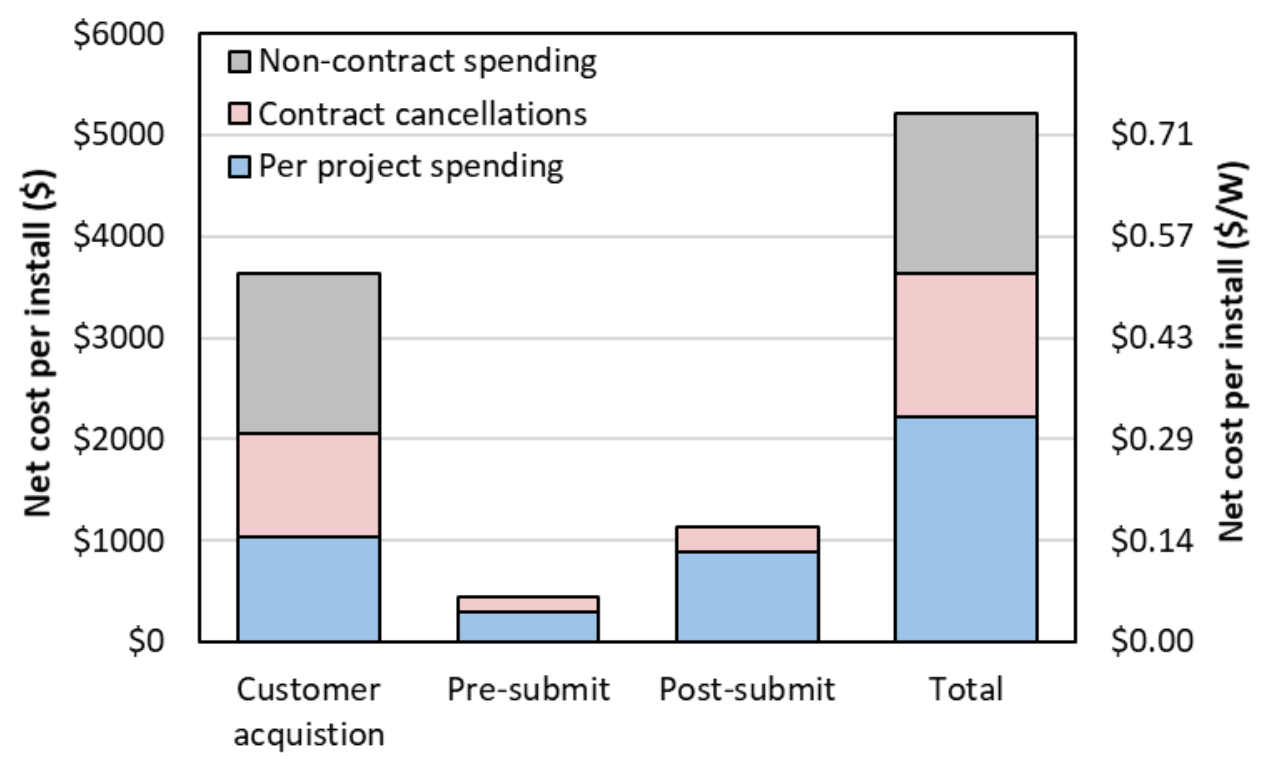

Figure 8. Average accumulation of net soft costs for installed projects by phase and cost type. Non-contract spending is from Feldman et al. (2021) and represents customer acquisition spending on potential customers who never sign contracts.

Altogether, this study finds that contract cancellations may account for about $25 \%$ of direct, preinstall soft costs for medium-to-large installers (i.e., $\$ 1,400$ of $\$ 5,200$ ). Moreover, lost spending on all unsuccessful customers (with and without contracts) potentially accounts for more than half of modeled soft costs. For some projects, these costs may be even higher, especially if cancellation rates, customer acquisition costs, and/or PII costs are higher than the modeled averages (i.e., at the higher end of the range in Figure 7). Higher cancellation rates in particular act as a multiplier of other costs, especially in earlier project phases where unsuccessful project rates are higher. 


\section{Conclusions}

This study is the first evaluation of U.S. residential solar PV cancellation rates, timelines, and cost impacts for medium-to-large installers. We utilize a project-level data set covering $10 \%$ of U.S. installs to assess when and how often contract cancellations occur. Critically, this data set includes both (1) cancelled and indefinitely stalled (idled) projects, and (2) unsuccessful projects from earlier phases in the post-contract, pre-install timeline (i.e., prior to PII application submission). Altogether, this data set gives a clearer accounting of unsuccessful projects following contract signing. Next, by leveraging NREL's soft cost model for residential solar, we estimate installer spending through each phase of the pre-install process. Applying our findings on cancellation timelines and rates, we then estimate the potential cost impacts to successful installs from contract cancellations.

Our work suggests that the rates and impacts of contract cancellations have been previously underestimated. Namely, we find that cancellations/unsuccessful projects:

- Are more common than previous estimates - on average, $51 \%$ of contracts in the data set fail to install PV systems, while the median installer cancellation rate was $33 \%$.

- Tend to occur earlier in project timelines, prior to PII application submission (73\% of cancelled projects), though a significant number (25\%) happen after permit/ATB approvals; only $2 \%$ of cancellations occur during application review.

- May contribute significantly to customer acquisition and other soft costs, with an average modeled cost impact of $\$ 0.20 / \mathrm{W}(\$ 1,400)$ and a median of $\$ 0.10 / \mathrm{W}(\$ 700)$ to successfully installed systems.

This study failed to find evidence of a correlation between longer or more variable PII timelines and cancellations, both for the entire data set and within individual AHJs. Variability in the data, especially in installer cancellation rates and across AHJs, masks any potential trends. Given the finding that only $1 \%-2 \%$ of project cancellations occur during the application review process itself, this study suggests that AHJ and/or utility review timelines alone are likely not a primary driver of cancellations. Future work should evaluate how other PII requirements and restrictions may impact cancellations and to what degree PII improvements can lower cancellation rates.

Contract cancellations were found to be a major contributor to soft costs, and these costs may be passed on to new customers who install PV systems. These findings also suggest that high cancellation rates/costs may help explain why soft costs remain high despite the dramatic decline in other PV costs over the last decade. However, more research is needed to identify how installers actually manage these cancellation costs and whether/how they are borne by new customers. Future work should also closely assess cost contributions from customer acquisition and early-phase cancellations.

This work focused exclusively on medium-to-large installers. Although the installers in our data together with those from SolarTRACE represent $22 \%-34 \%$ of the U.S. market, our results may not be representative for the smaller installers that make up much of the rest of the market. More 
research on these smaller installers is needed to evaluate their cancellation rates and costs in comparison to this study.

Finally, while the results of this study identified project phases when customers are more likely to cancel, we were not able to assess the reasons for cancellation. More work is needed to (1) evaluate why cancellations happen, especially for earlier- versus later-phase cancellations, and (2) understand whether these customers ultimately forgo solar completely or instead install with another company. Altogether, this future work offers opportunities to reduce cancellations, decrease soft costs, and improve the customer experience with solar PV installation. 


\section{References}

Burkhardt, J., Wiser, R., Darghouth, N., Dong, C.G., Huneycutt, J., 2015. Exploring the impact of permitting and local regulatory processes on residential solar prices in the United States. Energy Policy 78, 102-112. https://doi.org/10.1016/j.enpol.2014.12.020

Cook, J.J., Cruce, J., O’Shaughnessy, E., Ardani, K., Margolis, R., 2021. Exploring the link between project delays and cancelation rates in the U.S. rooftop solar industry. Energy Policy 156, 112421. https://doi.org/10.1016/j.enpol.2021.112421

Cox, M., 2020. H2 2020 U.S. solar PV system pricing. Wood Mackenzie.

Davis, M., White, B., Goldstein, R., Sun, X., Cox, M., Manghani, R., Rumery, S., Silver, C., Baca, J., 2021. Q2 2021 U.S. Solar Market Insight report. Wood Mackenzie \& Solar Energy Industries Association (SEIA).

Dong, C., Wiser, R., 2013. The impact of city-level permitting processes on residential photovoltaic installation prices and development times: An empirical analysis of solar systems in California cities. Energy Policy 63, 531-542. https://doi.org/10.1016/j.enpol.2013.08.054

Feldman, D., Ramasamy, V., Fu, R., Ramdas, A., Desai, J., Margolis, R., 2021. U.S. Solar Photovoltaic System and Energy Storage Cost Benchmark: Q1 2020. National Renewable Energy Laboratory (NREL), Golden, CO.

Hsu, J.H.-Y., 2018. Predictors for adoption of local solar approval processes and impact on residential solar installations in California cities. Energy Policy 117, 463-472.

https://doi.org/10.1016/j.enpol.2018.03.008

Inderst, R., Ottaviani, M., 2013. Sales Talk, Cancellation Terms and the Role of Consumer Protection. The Review of Economic Studies 80, 1002-1026.

https://doi.org/10.1093/restud/rdt005

Liao, Y., 2020. Weather and the Decision to Go Solar: Evidence on Costly Cancellations. Journal of the Association of Environmental and Resource Economists 7, 1-33.

https://doi.org/10.1086/705592

Moezzi, M., Ingle, A., Lutzenhiser, L., Sigrin, B.O., 2017. A Non-Modeling Exploration of Residential Solar Photovoltaic (PV) Adoption and Non-Adoption (No. NREL/SR--6A20-67727, 1379469). National Renewable Energy Laboratory (NREL), Golden, CO.

https://doi.org/10.2172/1379469

Mond, A., 2019. U.S. Residential PV Customer Acquisition Costs and Trends. Wood Mackenzie.

NREL, 2021. SolarTRACE [WWW Document]. solarapp.nrel.gov. URL https://solarapp.nrel.gov/solarTRACE (accessed 7.1.21). 
O'Shaughnessy, E., 2018. Trends in the market structure of US residential solar PV installation, 2000 to 2016: An evolving industry. Prog Photovolt Res Appl 26, 901-910.

https://doi.org/10.1002/pip.3030

O’Shaughnessy, E., Barbose, G., Wiser, R., 2020. Patience is a virtue: A data-driven analysis of rooftop solar PV permitting timelines in the United States. Energy Policy 144, 111615. https://doi.org/10.1016/j.enpol.2020.111615

O’Shaughnessy, E., Nemet, G.F., Pless, J., Margolis, R., 2019. Addressing the soft cost challenge in U.S. small-scale solar PV system pricing. Energy Policy 134, 110956. https://doi.org/10.1016/j.enpol.2019.110956

Parsons, S., Josefowitz, N., 2021. A No-Cost Rooftop Solar Stimulus. SPUR, San Francisco, CA.

Seel, J., Barbose, G.L., Wiser, R.H., 2014. An analysis of residential PV system price differences between the United States and Germany. Energy Policy 69, 216-226.

https://doi.org/10.1016/j.enpol.2014.02.022

Sinitskaya, E., Gomez, K.J., Bao, Q., Yang, M.C., MacDonald, E.F., 2019. Designing linked journey maps to understand the complexities of the residential solar energy market. Renewable Energy 145, 1910-1922. https://doi.org/10.1016/j.renene.2019.06.018

Stanfield, S., Kapla, K., McConnell, E.S., Haynes, R., Kooles, K., 2013. Minimizing Overlap in PV System Approval Processes. Interstate Renewable Energy Council (IREC).

Stanfield, S., Schroeder, E., Culley, T., 2012. Sharing Success: Emerging Approaches to Efficient Rooftop Solar Permitting. Interstate Renewable Energy Council (IREC).

White, B., 2021. U.S. PV Leaderboard: Year in Review 2020. Wood Mackenzie.

White, L.V., 2019. Increasing residential solar installations in California: Have local permitting processes historically driven differences between cities? Energy Policy 124, 46-53.

https://doi.org/10.1016/j.enpol.2018.09.034 


\section{Appendix A. Cancellation Cost Modeling}

The appendix presents the derivation of the overall cost increase equation along with a presentation and discussion of the cost and cancellation rate model scenarios.

\section{A.1 Modeled soft cost assumptions}

Table 5. Modeled Average Costs and Ranges for Each Phase and Cost Type

\begin{tabular}{|c|c|c|c|}
\hline Phase & Cost Type & $\begin{array}{l}\text { Average Cost } \\
\text { per Customer }\end{array}$ & Modeled Low/High Range (Rationale) \\
\hline \multirow[t]{4}{*}{$\begin{array}{l}\text { Customer } \\
\text { acquisition }\end{array}$} & \multirow[t]{2}{*}{$\begin{array}{l}\text { Advertising \& lead } \\
\text { generation* }\end{array}$} & \multirow[t]{2}{*}{$\$ 215$} & $\begin{array}{l}\$ 123 \text { (model low, e.g., managing } \\
\text { referrals) }\end{array}$ \\
\hline & & & $\$ 347$ (model high, e.g., direct marketing) \\
\hline & $\begin{array}{l}\text { Sales/qualification } \\
\text { (includes initial } \\
\text { engineering) }\end{array}$ & $\$ 496$ & NA \\
\hline & $\begin{array}{l}\text { Contract (sale } \\
\text { finalization) }\end{array}$ & $\$ 317$ & $\begin{array}{l}\$ 298 \text { (no financing) } \\
\$ 529 \text { (with financing) }\end{array}$ \\
\hline \multirow[t]{3}{*}{ Pre-submit } & Final system design & $\$ 163$ & NA \\
\hline & \multirow[t]{2}{*}{ Additional reviews } & \multirow[t]{2}{*}{$\$ 137$} & $\$ 0$ (no professional engineering reviews) \\
\hline & & & $\begin{array}{l}\$ 481 \text { (both structural and zoning } \\
\text { reviews) }\end{array}$ \\
\hline \multirow{12}{*}{$\begin{array}{l}\text { Application } \\
\text { review }\end{array}$} & \multirow{2}{*}{$\begin{array}{l}\text { Permit application \& } \\
\text { submission }\end{array}$} & \multirow[t]{2}{*}{$\$ 143$} & $\$ 28$ (online or mail/no driving) \\
\hline & & & $\begin{array}{l}\$ 305 \text { (multiple in-person trips, including } \\
\text { resubmit after corrections) }\end{array}$ \\
\hline & \multirow[t]{2}{*}{ Permit fee } & \multirow[t]{2}{*}{$\$ 475$} & $\$ 0$ (no permit fee) \\
\hline & & & $\$ 1,000$ (e.g., Williston Park village, NY) \\
\hline & \multirow[t]{2}{*}{$\begin{array}{l}\text { Interconnection/ATB } \\
\text { application \& submission }\end{array}$} & \multirow[t]{2}{*}{$\$ 20$} & $\begin{array}{l}\$ 0 \text { (no ATB, e.g., investor-owned utilities } \\
\text { in CA) }\end{array}$ \\
\hline & & & $\begin{array}{l}\$ 145 \text { (additional application research } \\
\text { required) }\end{array}$ \\
\hline & \multirow[t]{2}{*}{ Interconnection/ATB fee } & \multirow[t]{2}{*}{$\$ 95$} & $\$ 0$ (no ATB) \\
\hline & & & $\$ 500$ (e.g., City of Banning, CA) \\
\hline & \multirow{2}{*}{$\begin{array}{l}\text { Changes to permit } \\
\text { application }\end{array}$} & \multirow[t]{2}{*}{$\$ 110$} & $\$ 0$ (not required) \\
\hline & & & $\$ 143$ (required) \\
\hline & \multirow{2}{*}{$\begin{array}{l}\text { Changes to } \\
\text { interconnection } \\
\text { application }\end{array}$} & \multirow[t]{2}{*}{$\$ 45$} & $\$ 0$ (not required) \\
\hline & & & $\$ 126$ (required) \\
\hline $\begin{array}{l}\text { Post- } \\
\text { approval }\end{array}$ & Install & NA & $\begin{array}{l}\text { (Additional costs for install equipment, } \\
\text { planning, scheduling, etc. after } \\
\text { permit/interconnection approval not } \\
\text { modeled) }\end{array}$ \\
\hline
\end{tabular}

${ }^{*}$ Commissions and referral bonuses are only paid on successful projects and thus do not contribute to cancellation costs; as such, they are excluded from this study. 


\section{A.2 Cost Increase Equation Derivation}

Following the nomenclature from the report, let:

- $C_{S, p}=$ the spent cost per customer in phase $p(\$)$

- $\quad r_{p}=$ the unsuccessful project rate in phase $p(\%)$-i.e., the proportion of projects that start that phase, with costs spent on them in that phase, but that do not ultimately install PV systems

- $\left(1-r_{p}\right)=$ successful project rate in phase $p(\%)$, also known as the conversion rate for that phase

- $\Delta N C_{P}=$ Cost increase per install, or the additional cost lost for spending on unsuccessful projects that must be borne by successful installs (\$).

The total spending in a given phase $\left(N C_{p, N}\right)$ for $N$ projects that start that phase is:

$$
N C_{p, N}=N\left(r_{p} C_{S, p}+\left(1-r_{p}\right) C_{S, p}\right)
$$

The $r C_{S, p}$ term is the spending lost to cancellations (\$), while the $\left(1-r_{p}\right) C_{S, p}$ term is the spending on successfully installed projects. Spending on successful installs happens regardless of whether or how many other projects are cancelled, so that term can be excluded from the cost increase. Thus, the cost increase is simply the total lost spending (\$) divided across all successful installs, namely:

$$
\Delta N C_{p}=\frac{N r_{p} C_{S, p}}{N\left(1-r_{p}\right)}=\frac{r_{p} C_{S, p}}{\left(1-r_{p}\right)}
$$

This calculation can be performed across all of the cost stages in the cost model, using the given $r_{p}$ and $C_{S, p}$ for each stage. Thus, the total cost increase is the summation function given in the methods section:

$$
\Delta N C_{P}=\sum_{p=1}^{P} \frac{r_{p}}{1-r_{p}} C_{S, p}
$$

\section{A.3 Cost and Cancellation Rate Model Assumptions and Discussion}

Calculations to estimate the cost increase due to unsuccessful projects utilized three spent cost scenarios and four unsuccessful project rate levels, all based on the results from this work. The three spent cost scenarios, namely, the average cost per customer and the low and high range values, are taken directly from Figure 6 . The four unsuccessful project rate levels are simplified from Table 3 and include the average (50\% overall rate), low (20\%), high (55\%), and median $(35 \%)$ values. These scenarios were combined to give both best-case and worst-case results, along with two possible middle results (i.e., a higher average and a lower median). Table 6 shows the combination between the cost and cancel rate scenarios used, along with the unsuccessful project rates at each major phase of the pre-install process. 
Table 6. Cost and Cancel Rate Scenario Combinations Utilized To Estimate the Cost Increase Due to Unsuccessful Projects, Along With the Unsuccessful Project Rate at Each Major Phase of the Pre-Install Process

\begin{tabular}{cccccc}
\hline \multicolumn{2}{c}{ Cost Level Scenario } & Low & Average & Average & High \\
\hline \multicolumn{2}{c}{ Cancel Rate Scenario } & Low & Median & Average & High \\
Cancel Rate & Pre-submit & $20 \%$ & $35 \%$ & $50 \%$ & $55 \%$ \\
& Application review & $8 \%$ & $11 \%$ & $22 \%$ & $25 \%$ \\
& Post-approval & $6 \%$ & $9 \%$ & $20 \%$ & $24 \%$ \\
\hline
\end{tabular}

The cancellation rate scenarios only define the unsuccessful project rates at the start of each of the major pre-install phases. Thus, rates must be assumed for cost stages within the pre-submit and review phases. First, rates were assumed to remain constant throughout the entire review phase until approval, given the low cancellation rate in this phase and the lack of data about any rate changes at the "changes to the permit/interconnection" stage. No costs were modeled between permit approval and installation (purchased equipment is assumed to be reused for successful installs, etc.), and cancellation rates after installation are negligibly low and not considered here. Customer acquisition phases only included costs for projects that successfully signed contracts (excluding spending on potential customers that never signed contracts).

The modeled unsuccessful project rates decreased between the "contract signing" and "permit fill/submit" stages, as seen in Figure 9. Two scenarios for this decrease were considered: a linear decrease (via interpolation), and an approximately quadratic/exponential decrease, with a 50\% decrease between contract signing and final engineering followed by a $25 \%$ decrease to the additional reviews stage (i.e., to $12.5 \%$ of the rate difference between contract signing and permit submission). The quadratic scenario is shown in Figure 9. The overall cost increase results were found to be slightly higher for the linear scenarios due to higher cancellation rates throughout the system design and additional review stages. However, given the lack of data on actual rate changes during the pre-submit stage, the lower cost (quadratic) scenario is presented in the results in the report (Table 4). The comparison between the linear and quadratic scenario results is shown in Table 7. 


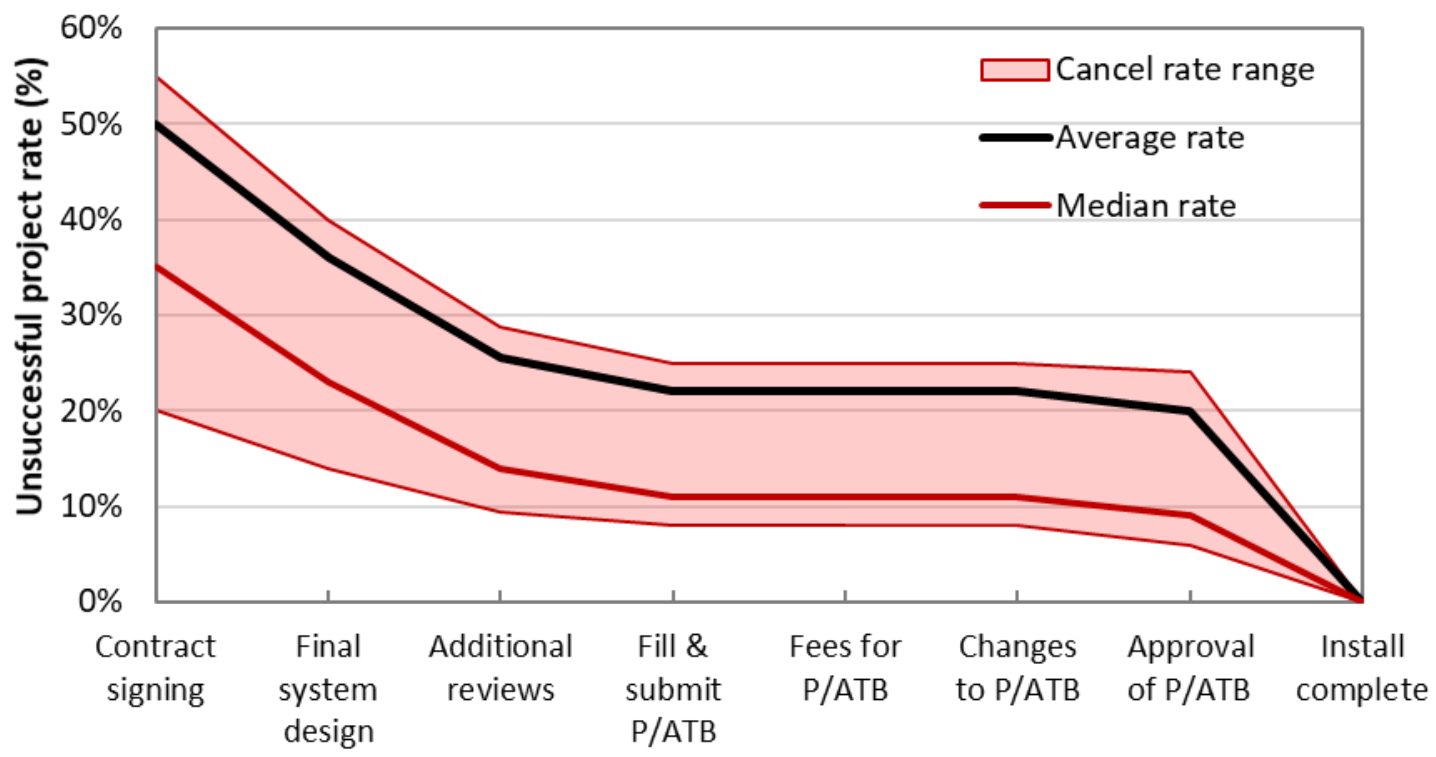

Figure 9. Cancellation rate model utilized for the results in the report, i.e., using an approximately quadratic decrease in unsuccessful project rates between contract signing and permit submission.

Table 7. Cost Increase Results by Unsuccessful Project Rate Scenario

\begin{tabular}{|c|c|c|c|c|c|}
\hline Scenario & $\begin{array}{c}\text { Average } \\
\text { Range } \\
\text { (Median) }\end{array}$ & $\begin{array}{l}\text { Customer } \\
\text { Acquisition }\end{array}$ & Pre-Submit & Post-Submit & $\begin{array}{c}\text { Total } \\
\text { (Post-Contract) }\end{array}$ \\
\hline \multirow[t]{2}{*}{$\begin{array}{l}\text { Quadratic } \\
\text { Decrease }\end{array}$} & $\begin{array}{l}\text { Net cost per } \\
\text { install }\end{array}$ & $\begin{array}{c}\$ 1,030 \\
\$ 230-\$ 1,680 \\
(\$ 550)\end{array}$ & $\begin{array}{c}\$ 140 \\
\$ 30-\$ 300 \\
(\$ 70)\end{array}$ & $\begin{array}{c}\$ 250 \\
\$ 3-\$ 740 \\
(\$ 110)\end{array}$ & $\begin{array}{c}\$ 1,420 \\
\$ 260-\$ 2,720 \\
(\$ 730)\end{array}$ \\
\hline & Net $\$ / W$ & $\begin{array}{c}\$ 0.147 \\
\$ 0.033-\$ 0.240 \\
(\$ 0.079)\end{array}$ & $\begin{array}{c}\$ 0.020 \\
\$ 0.004-\$ 0.043 \\
(\$ 0.010)\end{array}$ & $\begin{array}{c}\$ 0.036 \\
\$ 0-\$ 0.106 \\
(\$ 0.016)\end{array}$ & $\begin{array}{c}\$ 0.202 \\
\$ 0.037-\$ 0.388 \\
(\$ 0.105)\end{array}$ \\
\hline \multirow[t]{2}{*}{$\begin{array}{l}\text { Linear } \\
\text { Decrease }\end{array}$} & $\begin{array}{l}\text { Net cost per } \\
\text { install }\end{array}$ & Same & $\begin{array}{c}\$ 170 \\
\$ 30-\$ 390 \\
(\$ 90)\end{array}$ & Same & $\begin{array}{c}\$ 1450 \\
\$ 260-\$, 2810 \\
(\$ 760)\end{array}$ \\
\hline & Net $\$ / \mathrm{W}$ & Same & $\begin{array}{c}\$ 0.025 \\
\$ 0.004-\$ 0.056 \\
(\$ 0.013)\end{array}$ & Same & $\begin{array}{c}\$ 0.207 \\
\$ 0.038-\$ \$ 0.401 \\
(\$ 0.108)\end{array}$ \\
\hline
\end{tabular}

